\title{
Construction of Supported Organometallics Using Cycloplatinated Arylamine Ligands
}

\author{
Michel D. Meijer, ${ }^{\dagger}$ Arjan W. Kleij, ${ }^{\dagger}$ B. Scott Williams, ${ }^{\dagger}$ Dianne Ellis, ${ }^{\ddagger}$ \\ Martin Lutz, ${ }^{\ddagger}$ Anthony L. Spek, ${ }^{\ddagger} \S$ Gerard P. M. van Klink, ${ }^{\dagger}$ and \\ Gerard van Koten*,t
}

Department of Metal-Mediated Synthesis, Debye Institute, Utrecht University, Padualaan 8, $3584 \mathrm{CH}$ Utrecht, The Netherlands, and Department of Crystal and Structural Chemistry, Bijvoet Center for Biomolecular Research, Utrecht University, Padualaan 8, $3584 \mathrm{CH}$ Utrecht, The Netherlands

Received J une 11, 2001

The preparation of ortho-chelating aminoaryl ligands $\left(\left[\mathrm{C}_{6} \mathrm{H}_{3}\left(\mathrm{CH}_{2} \mathrm{NMe}_{2}\right)-2-\mathrm{R}-4\right]^{-}\right.$, abbreviated as $\mathrm{C}, \mathrm{N}$ ) containing a pendant hydroxymethyl group is described. These ligands have been cycloplatinated with cis- $\mathrm{PtCl}_{2}(\mathrm{DMSO})_{2}$, yielding the corresponding $\mathrm{C}, \mathrm{N}$-platinum(II) complexes. The pendant hydroxymethyl substituent is a versatile group for attachment of the organometallic moiety to macromolecules, which has been demonstrated by attaching the $\mathrm{C}, \mathrm{N}$-platinum complexes to a dendritic wedge and to $\mathrm{C}_{60}$.

\section{Introduction}

One of the most rapidly developing fields in organometallic chemistry is the construction of supramolecular structures $^{1}$ and organometallic assemblies. ${ }^{2}$ In some cases, the macromolecular structures contain (metal) sites or functional groups that can lead to new materials, applicable in host-guest chemistry ${ }^{3}$ and catalysis ${ }^{4}$ or as sensors. ${ }^{5} \mathrm{In}$ the construction of these materials, organometallic moieties have been introduced via a ligand modification/immobilization and subsequent metalation step, which is not al ways the optimal procedure. For example, we have encountered unexpected interand intramolecular complexation behavior of C,N-derivatized carbosilane dendrimers $(\mathrm{C}, \mathrm{N}=\mathrm{C}, \mathrm{N}$-chel ating $\left.\left[\mathrm{C}_{6} \mathrm{H}_{3}\left(\mathrm{CH}_{2} \mathrm{NMe}_{2}\right)-2-\mathrm{R}-4\right]^{-}\right)$during metalation steps, resulting in the formation of multimetallic aggregates. ${ }^{6}$

An approach to circumvent these interactions involves the attachment of a compl ete organometallic moi ety to a macromolecular structure or to a support. This has been successfully demonstrated with supports such as silica surfaces, ${ }^{7}$ (hyperbranched) polymers, ${ }^{8}$ dendrimers, ${ }^{9}$ and more recently, for the functionalization of the periphery of dendrimers with NCN-palladium(II) and

* To whom correspondence should be addressed. E-mail: g.vankoten@ chem.uu.nl. Fax: +(31) 302523615.

+ Debye Institute.

* Bijvoet Center for Biomolecular Research.

$\S$ Address correspondence pertaining to crystallographic studies to this author. E-mail: a.I.spek@chem.uu.nl.

(1) Lehn, J .-M. Angew. Chem., Int. Ed. Engl. 1990, 29, 1304.

(2) (a) Stang, P. J .; Olenyuk, B. Acc. Chem. Res. 1997, 30, 502. (b) Leininger, S.; Olenyuk, B.; Stang, P. J . Chem. Rev. 2000, 100, 853.

(3) Vögtle, F. ComprehensiveSupramolecular Chemistry; Pergamon/ Elsevier Press: New York, 1997; Vol. 2.

(4) (a) Sanders, J. K. M. Chem. Eur. J. 1998, 4, 1378. (b) Kirby, A. J. Angew. Chem., Int. Ed. Engl. 1996, 35, 707.

(5) (a) Albrecht, M.; Gossage, R. A.; Spek, A. L.; van Koten, G. Chem Commun. 1998, 1003. (b) Albrecht, M.; van Koten G. Adv. Mater. 1999, 11, 171. (c) Albrecht, M.; Lutz, M.; Spek, A. L.; van Koten, G. Nature 2000, 406, 970.

(6) (a) Kleij, A. W.; Kleijn, H.; J astrzebski, J. T. B. H.; Smeets, W. J. J .; Spek, A. L.; van Koten, G. Organometallics 1999, 18, 268. (b) Kleij, A. W.; Klein Gebbink, R. J . M.; van den Nieuwenhuijzen, P. A. J.; Kooiman, H.; Lutz, M.; Spek, A. L.; van Koten, G. Organometallics 2001, 20, 634.
-platinum(II) complexes ( $\mathrm{NCN}=\left[\mathrm{C}_{6} \mathrm{H}_{2}\left(\mathrm{CH}_{2} \mathrm{NMe}_{2}\right)_{2}-2,6-\right.$ $\mathrm{R}-4]^{-}$), yiel ding macromolecular catalysts and $\mathrm{SO}_{2-}$ sensing dendritic macromolecules, respectively.5,10 To devel op this approach for the C,N-ligand, we have studied the synthesis and structural characterization of a series of cycloplatinated $\mathrm{C}, \mathrm{N}$-derived ligands containing a pendant hydroxymethyl group (para to the $\mathrm{CH}_{2} \mathrm{NMe}_{2}$ substituent). This group serves as a suitable linker for attachment of the organometallic fragment to the macromol ecular framework. In this fashion, C,N-platinum(II) complexes have been anchored to a Fréchet-G1 dendron and to $\mathrm{C}_{60}$.

\section{Results and Discussion}

Synthesis and Characterization of Cycloplatinated $\mathbf{C}, \mathbf{N}-\mathbf{C}$ omplexes. Synthetic protocols were developed for cycloplatinated $\mathrm{C}, \mathrm{N}$-complexes containing a hydroxymethyl group, which can be used as a linker to attach the organoplatinum fragment to a macromolecular structure. 4-[(Dimethylamino)methyl)]benzal dehyde was reacted with $\mathrm{LiAlH}_{4}$, affording benzyl alcohol $\mathbf{1}$ (Scheme 1). Direct cycloplatination of $\mathbf{1}$ with an equimolar amount of cis- $\mathrm{PtCl}_{2}(\mathrm{DMSO})_{2}$ in the presence of $\mathrm{NaOAc}$ yielded the platinum DMSO derivative 2 , which was converted into the corresponding triphenylphosphine derivative 3 via ligand exchange.

To double the number of organometallic units per hydroxyl linking group, the bis $(\mathrm{H}-\mathrm{C}, \mathrm{N})$-ligand $\mathbf{4}$ has also been prepared (Scheme 2). Treatment of 4-[(dimethylamino)methyl)]benzaldehyde with an equimolar amount

(7) (a) Price, P. M.; Clark, J . H.; Macquarrie, D. J . J . Chem. Soc., Dalton Trans. 2000, 101. (b) Macquarrie, D. J .; Fairfield, S. E. J . Mater. Chem. 1997, 7, 2201. (c) van de Kuil, L. A.; Grove, D. M.; Zwikker, J . W.; J enneskens, L. W.; Drenth, W.; van Koten, G. Chem. Mater. 1996, 6, 1675.

(8) Schlenk, C.; Kleij, A. W.; Frey, H.; van Koten, G. Angew. Chem., Int. Ed. 2000, 39, 3445.

(9) F or reviews on this subject, see: (a) van Koten, G.; J astrzebski, J . T. B. H.J . Mol. Catal. A 1999, 146, 317. (b) Majoral, J . P.; Caminade, A.-M. Chem. Rev. 1999, 99, 845. (c) Newkome, G. R.; He, E.; Moorefield, C. N. Chem. Rev. 1999, 99, 1698. (d) Bosman, A. W.; J anssen, H. M.; Meijer, E. W. Chem. Rev. 1999, 99, 1665.

(10) Dijkstra, H. P.; Albrecht, M.; Ronde, N.; van Klink, G. P. M.; Vogt, D.; van Koten, G., to be published. 
Scheme 1. Synthesis of $3^{a}$
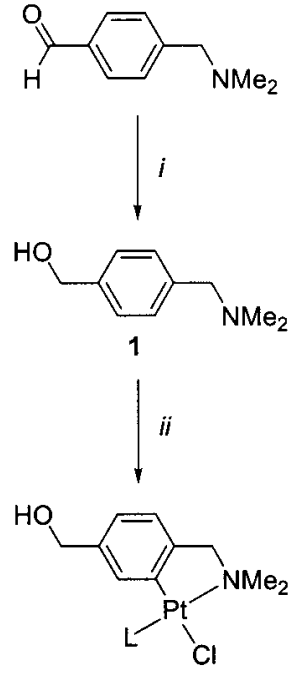

$2 \mathrm{~L}=\mathrm{DMSO}$
$3 \mathrm{~L}=\mathrm{PPh}_{3}$

a Reagents and Conditions: (i) $\mathrm{LiAlH}_{4}, \mathrm{Et}_{2} \mathrm{O}, 0{ }^{\circ} \mathrm{C}$; (ii) cis$\mathrm{PtCl}_{2}(\mathrm{DMSO})_{2}, \mathrm{NaOAC}, \mathrm{MeOH}, 65^{\circ} \mathrm{C}$; (iii) $\mathrm{PPh}_{3}, \mathrm{CH}_{2} \mathrm{Cl}_{2}$.

Scheme 2. Synthesis of $6^{a}$<smiles>CN(C)Cc1ccc(C=O)cc1</smiles>

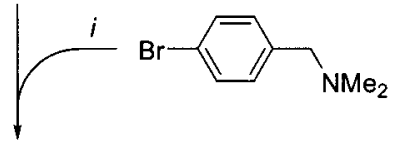<smiles>CNCc1ccc(C(O)c2ccc(CN(C)C)cc2)cc1</smiles>

4

ii<smiles>CN(C)[P](Cl)(Cl)c1cc(C(O)c2ccc3c(c2)P(C)(Cl)(I)NC3)ccc1CN</smiles><smiles>CCCOc1ccccc1-c1ccccc1</smiles>

aReagents and Conditions: (i) 2 equiv of $\mathrm{t}-\mathrm{BuLi}, \mathrm{Et}_{2} \mathrm{O},-78$ ${ }^{\circ} \mathrm{C}$ to rt; (ii) 2 equiv of cis- $\mathrm{PtCl}_{2}(\mathrm{DMSO})_{2}, 2$ equiv of $\mathrm{NaOAC}$ $\mathrm{MeOH}, 65^{\circ} \mathrm{C}$; (iii) $2 \mathrm{PPh}_{3}, \mathrm{CH}_{2} \mathrm{Cl}_{2}$.

of the lithium reagent $\left[\mathrm{Li}\left\{\mathrm{C}_{6} \mathrm{H}_{4}\left(\mathrm{CH}_{2} \mathrm{NMe}_{2}\right)-4\right\}\right]$ in diethyl ether yielded the bis(H-C,N)-functionalized derivative 4. The introduction of a platinum center in each of the two $\mathrm{C}, \mathrm{N}$-sites was achieved using the same methodology as described for $\mathbf{2}$, producing the bisplatinum compound $\mathbf{5}$ as a white solid. Subsequent treatment of $\mathbf{5}$ with $\mathrm{PPh}_{3}$ in $\mathrm{CH}_{2} \mathrm{Cl}_{2}$ afforded bisphosphine complex 6 .

The platinum complexes $\mathbf{3}$ and $\mathbf{6}$ were characterized by NMR spectroscopy ( ${ }^{1} \mathrm{H}$ (Table 1$),{ }^{13} \mathrm{C}\left\{{ }^{1} \mathrm{H}\right\}$, and ${ }^{31} \mathrm{P}$ ) and elemental analysis. Remarkably high field shifts of the pendant $\mathrm{OH}$ groups were observed by ${ }^{1} \mathrm{H}$ NMR spectroscopy $\left(\mathrm{CDCl}_{3}\right.$, Table 1$)$. These shifts can be
Table 1. Selected ${ }^{1} H$ NMR Data,b for $1-6$

\begin{tabular}{ccclll}
\hline complex & $\mathrm{H}_{\text {ortho }}$ & aryl-H & \multicolumn{1}{c}{$\mathrm{CH}_{2} \mathrm{~N}$} & $\mathrm{NMe}_{2}$ & $\mathrm{OH}$ \\
\hline $\mathbf{1}$ & & $7.31,7.26$ & 3.40 & 2.20 & 3.06 \\
$\mathbf{2}$ & 7.92 & $7.11,7.06$ & 3.98 & 2.90 & 1.70 \\
$\mathbf{3}$ & 6.34 & $7.04,7.82$ & 3.96 & 2.97 & 0.68 \\
$\mathbf{4}$ & & $7.29,7.20$ & 3.34 & 2.13 & 5.08 \\
$\mathbf{5}$ & 8.03 & $7.13,6.99$ & $3.94 / 3.91^{\mathrm{c}}$ & $2.98 / 2.88$ & 2.29 \\
$\mathbf{6}$ & 6.43 & $6.70,5.90$ & $4.02 / 3.98^{\mathrm{c}}$ & $2.97 / 2.95$ & 0.52
\end{tabular}

a $300 \mathrm{MHz}_{2} \mathrm{CDCl}_{3}, \delta$ in ppm. bPlatinum and phosphorus couplings not included. ${ }^{\mathrm{C}} \mathrm{AB}$ system.

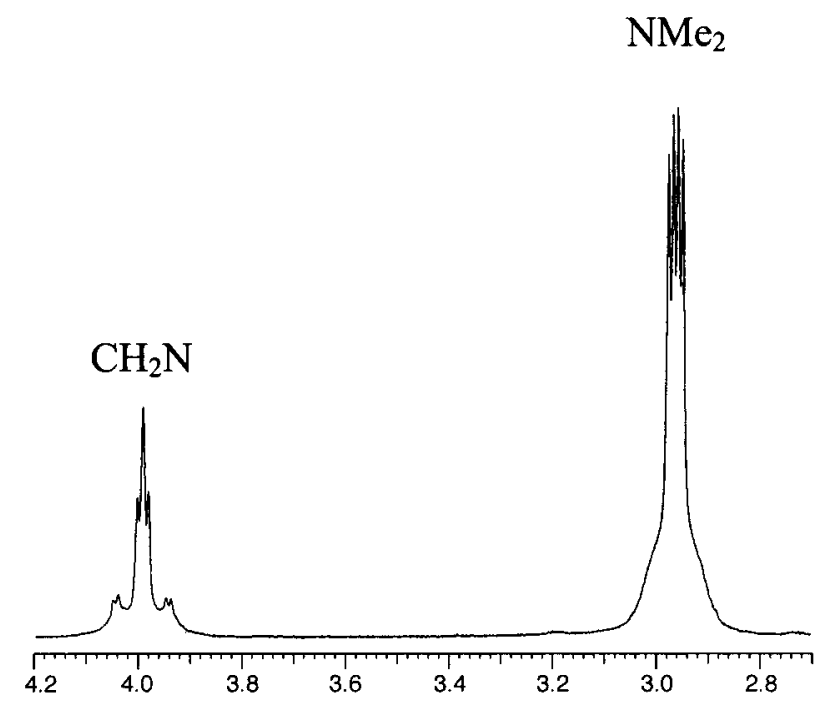

Figure 1. Part of the ${ }^{1} \mathrm{H}$ NMR spectrum of $\mathbf{6}$, showing the resonances for the benzylic and $\mathrm{NMe}_{2}$ protons. ${ }^{11}$

attributed to intramolecular interaction with the DMSO and $\mathrm{PPh}_{3}$ ligands. Similar behavior was observed for the aryl protons of the platinum complexes, which also shifted to higher field with respect to the signals in the parent ligands.

For the bis-C,N complexes 5 and $\mathbf{6}$, two resonances with platinum (and phosphorus for 6) couplings were observed for the $\mathrm{NMe}_{2}$ groups. The resonances for the protons on the benzylic positions (donor arms) were observed as AB patterns (Figure 1), which reflects the stereogenicity of the para substituent. ${ }^{11}$ This pattern was observed not only for $\mathbf{5}$ and $\mathbf{6}$ but also for all complexes derived from 6 (see below). ${ }^{195} \mathrm{Pt}-{ }^{31} \mathrm{P}$ couplings of $4261 \mathrm{~Hz}$ (3) and $4281 \mathrm{~Hz}$ (6), respectively, were observed by ${ }^{31} \mathrm{P}\left\{{ }^{1} \mathrm{H}\right\}$ NMR spectroscopy. These data are consistent with the depicted structures of $\mathbf{3}$ and $\mathbf{6}$, i.e., with the $\mathrm{PPh}_{3}$ ligand trans to the nitrogen donor atom.

Compound $\mathbf{6}$ was further characterized by a single crystal structure determination. The molecular structure of $\mathbf{6}$ in the solid state (Figure 2) comprises two platinum(I I) metal centers, which each have a distorted square planar environment (Table 2), with $\mathrm{C}, \mathrm{N}$-bite angles of $80.8(2)^{\circ}$ and $81.2(2)^{\circ}$. The substituents $\mathrm{O}(1)$ and $\mathrm{H}(10)$ at carbon atom $\mathrm{C}(10)$ are positionally disordered (pseudo-2-fold rotation axis). Only the major disorder component (54.7\% occupancy) is shown in Figure 2. No hydrogen-bonding interaction was observed for 6 in the solid state between the $\mathrm{C}(\mathrm{H}) \mathrm{OH}$ and $\mathrm{Pt}-\mathrm{Cl}$ groupings such as has been observed for the corre sponding complexes $\left[\mathrm{PtCl}\left(\mathrm{C}_{6} \mathrm{H}_{2}\left\{\mathrm{CH}_{2} \mathrm{NMe}_{2}\right\}_{2}-2,6-\mathrm{OH}-4\right)\right]^{5}$ and $\left[\mathrm{PtCl}\left(\mathrm{C}_{6} \mathrm{H}_{2}\left\{\mathrm{CH}_{2} \mathrm{NMe}_{2}\right\}_{2}-2,6-\left\{\mathrm{CH}_{2} \mathrm{OH}\right\}-4\right)\right]{ }^{12}$

Derivatization of $\mathbf{3}$ and $\mathbf{6}$. The organometallic fragments $\mathbf{3}$ and $\mathbf{6}$ were prepared for use as precursors in the synthesis of larger macromolecular structures. 


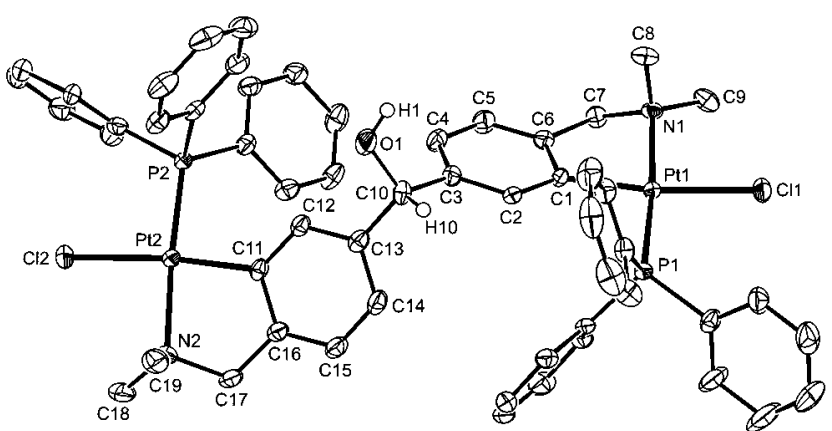

Figure 2. Displacement ellipsoid plot ( $50 \%$ probability level) of the molecular structure of $\mathbf{6}$, with the adopted numbering scheme. All hydrogen atoms except $\mathrm{H}(1)$ and $\mathrm{H}(10)$ have been omitted for clarity. The substituents $\mathrm{O}(1)$ and $\mathrm{H}(10)$ at carbon atom $\mathrm{C}(10)$ are positionally disordered (pseudo-2-fold rotation axis). Only the major disorder component $(54.7 \%$ occupancy) is shown.

Table 2. Selected Bond Distances $(\AA)$ and Angles (deg) of 6

\begin{tabular}{llll}
\hline $\mathrm{Pt}(1)-\mathrm{Cl}(1)$ & $2.3917(12)$ & $\mathrm{Pt}(2)-\mathrm{Cl}(2)$ & $2.3856(11)$ \\
$\mathrm{Pt}(1)-\mathrm{P}(1)$ & $2.2270(11)$ & $\mathrm{Pt}(2)-\mathrm{P}(2)$ & $2.2282(12)$ \\
$\mathrm{Pt}(1)-\mathrm{N}(1)$ & $2.143(4)$ & $\mathrm{Pt}(2)-\mathrm{N}(2)$ & $2.164(4)$ \\
$\mathrm{Pt}(1)-\mathrm{C}(1)$ & $2.025(4)$ & $\mathrm{Pt}(2)-\mathrm{C}(2)$ & $2.016(4)$ \\
$\mathrm{Cl}(1)-\mathrm{Pt}(1)-\mathrm{P}(1)$ & $93.08(4)$ & $\mathrm{Cl}(2)-\mathrm{Pt}(2)-\mathrm{P}(2)$ & $92.35(4)$ \\
$\mathrm{Cl}(1)-\mathrm{Pt}(1)-\mathrm{N}(1)$ & $91.59(11)$ & $\mathrm{Cl}(2)-\mathrm{Pt}(2)-\mathrm{N}(2)$ & $91.56(11)$ \\
$\mathrm{P}(1)-\mathrm{Pt}(1)-\mathrm{C}(1)$ & $94.39(13)$ & $\mathrm{P}(2)-\mathrm{Pt}(2)-\mathrm{C}(11)$ & $94.98(13)$ \\
$\mathrm{N}(1)-\mathrm{Pt}(1)-\mathrm{C}(1)$ & $80.85(17)$ & $\mathrm{N}(2)-\mathrm{Pt}(2)-\mathrm{C}(11)$ & $81.24(17)$ \\
$\mathrm{P}(1)-\mathrm{Pt}(1)-\mathrm{N}(1)$ & $175.04(11)$ & $\mathrm{P}(2)-\mathrm{Pt}(2)-\mathrm{N}(2)$ & $175.50(11)$ \\
$\mathrm{Cl}(1)-\mathrm{Pt}(1)-\mathrm{C}(1)$ & $171.87(13)$ & $\mathrm{Cl}(2)-\mathrm{Pt}(2)-\mathrm{C}(11)$ & $172.11(13)$ \\
interplanar angle $\mathrm{Pt}(1)-\mathrm{Cl}(1)-\mathrm{P}(1)$ and $\mathrm{Pt}(1)-\mathrm{N}(1)-\mathrm{C}(1)$ & $3.42(18)$ \\
& & $\mathrm{Pt}(2)-\mathrm{Cl}(2)-\mathrm{P}(2)$ and $\mathrm{Pt}(1)-\mathrm{N}(1)-\mathrm{C}(1)$ & $3.98(17)$
\end{tabular}

The pendant hydroxymethyl group in the platinum complexes $\mathbf{3}$ and $\mathbf{6}$ is well suited for direct reaction with acid chloride-substituted dendrimers or dendrons. More over, reaction with malonyl chloride enables coupling to $\mathrm{C}_{60}$ using the Bingel cyclopropanation reaction. ${ }^{13} \mathrm{To}$ demonstrate the use of $\mathrm{C}, \mathrm{N}$-platinum complexes in the synthesis of macromolecular structures based on dendrimers, the $\mathrm{C}, \mathrm{N}$-platinum complex $\mathbf{3}$ was attached to a small Fréchet type dendron/wedge. ${ }^{14}$ Addition of [G1]$\mathrm{C}(\mathrm{O}) \mathrm{Cl}$ to an equimolar amount of 3 in $\mathrm{CH}_{2} \mathrm{Cl}_{2}$ in the presence of base $\left(\mathrm{NEt}_{3}\right)$ yielded the dendritic platinum(II) complex 7 in $65 \%$ isolated yield (Scheme 3). Conclusive evidence for the formation of $\mathbf{7}$ was provided by the disappearance of the doublet resonance assigned to the benzylic protons of the hydroxymethyl group in $\mathbf{3}$ $(\delta=3.96)$ and the appearance of a new singlet resonance at $4.62 \mathrm{ppm}$, attributed to the methylene group of the newly formed ester linkage. Furthermore, MALDITOF mass spectrometric analysis displayed the molecular ion peak $[\mathrm{M}]^{+}$and a $[\mathrm{M}-\mathrm{Cl}]^{+}$peak for $\mathrm{m} / \mathrm{z} 972.82$ and 938.07 , respectively. Similar ${ }^{195} \mathrm{Pt}-{ }^{31} \mathrm{P}$ coupling constants were observed for $3(\mathrm{~J}=4261 \mathrm{~Hz}$ ) and for the [G1] dendrimer complex $7(\mathrm{~J}=4235 \mathrm{~Hz})$. This

(11) Compounds 4-6 (and their derivatives) are interesting cases of an achiral $X Y C R_{2}$ system where the C-center is prochiral, but any prochiral center in $\mathrm{R}$ is diastereotopic. For $\mathbf{4}$, only singlets were observed by ${ }^{1} \mathrm{H} N \mathrm{NMR}$, most likely due to a too small $\Delta \delta$ for the benzylic protons and $\mathrm{NMe}_{2}$ groups.

(12) Slagt, M. Q.; van de Coevering, R.; Klein Gebbink, R. J . M.; Lutz, M.; Spek, A. L., van Koten, G., unpublished results.

(13) See for instance: (a) Bingel, C. Chem. Ber. 1993, 126, 1957. (b) Nierengarten, J .-F .; Felder, D.; Nicoud, J .-F. Tetrahedron Lett. 1998, 2747, and references therein.

(14) (a) Hawker, C. J .; Fréchet, J . M. J . J . Am. Chem. Soc. 1990 112, 7638. (b) Wool ey, K. L.; Hawker, C. J .; Fréchet, J. M. J. J . Am Chem. Soc. 1990, 112, 4253. (c) Albrecht, M.; Hovestad, N.J .; Boersma, J .; van Koten, G. Chem. Eur. J . 2001, 7, 1289.
Scheme $3^{a}$

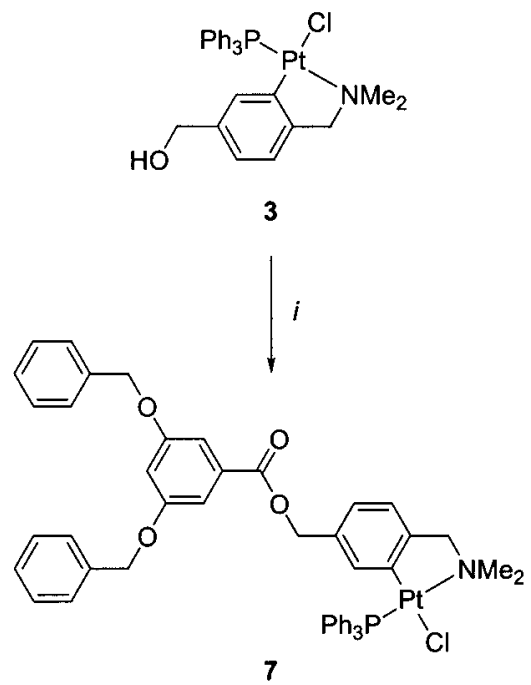

a Reagents and Conditions: (i) [G1]-C(O)Cl, $\mathrm{NEt}_{3}, \mathrm{CH}_{2} \mathrm{Cl}_{2}$.

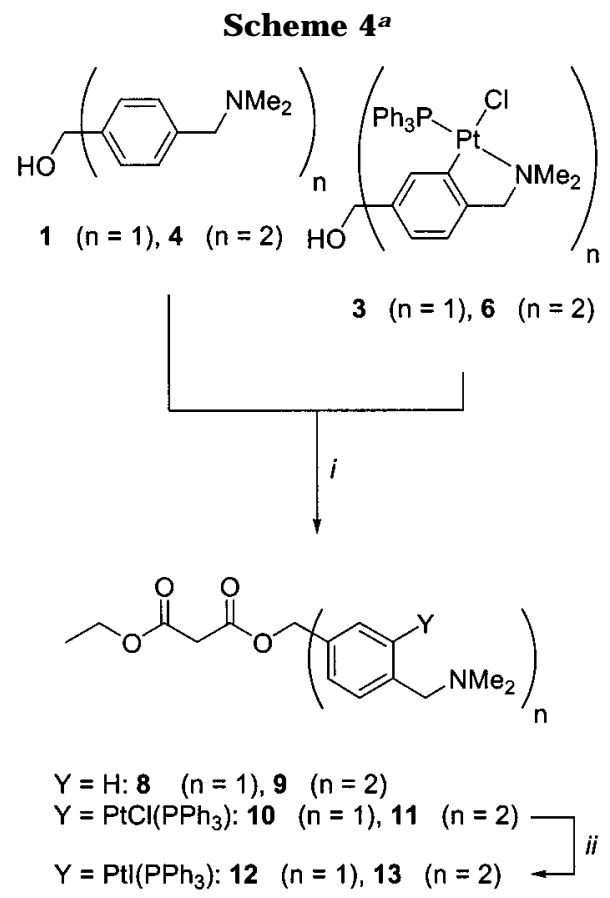

a Reagents and Conditions: (i) ethyl malonyl chloride, pyridine, or $\mathrm{NEt}_{3}, \mathrm{Et}_{2} \mathrm{O}, 0^{\circ} \mathrm{C}$; (ii) Nal, acetone.

suggests an unchanged spatial arrangement of the ligands about the platinum center. Moreover, the highyield synthesis of $\mathbf{7}$ and its purity demonstrate the stability of the cycloplatinated C,N-building block.

Synthesis of Fullerene Derivatives. Methanofullerene $\mathrm{C}, \mathrm{N}$-platinum complexes are accessible via two routes: (a) preparation of the methanofullerene ligand followed by transmetalation, or (b) direct cyclopropanation of $\mathrm{C}_{60}$ with a malonate $\mathrm{C}, \mathrm{N}$-platinum complex. Both routes were studied for comparison. For this purpose, ethyl malonyl tails were attached not only to the C,N-platinum complexes $\mathbf{3}$ and $\mathbf{6}$ but also to the parent $\mathrm{C}, \mathrm{N}$-ligands $\mathbf{1}$ and $\mathbf{4}$. Reaction of $\mathbf{1}, \mathbf{3}, \mathbf{4}$, and $\mathbf{6}$ with ethyl malonyl chloride in $\mathrm{Et}_{2} \mathrm{O}$ in the presence of base ( $\mathrm{NEt}_{3}$ or pyridine) at $0{ }^{\circ} \mathrm{C}$ afforded the corresponding malonate compounds 8-11 (Scheme 4). To avoid halogen scrambling (i.e, chloride-iodide exchange by $\mathrm{I}_{2}$ during the Bingel reaction of the platinum-chloride 


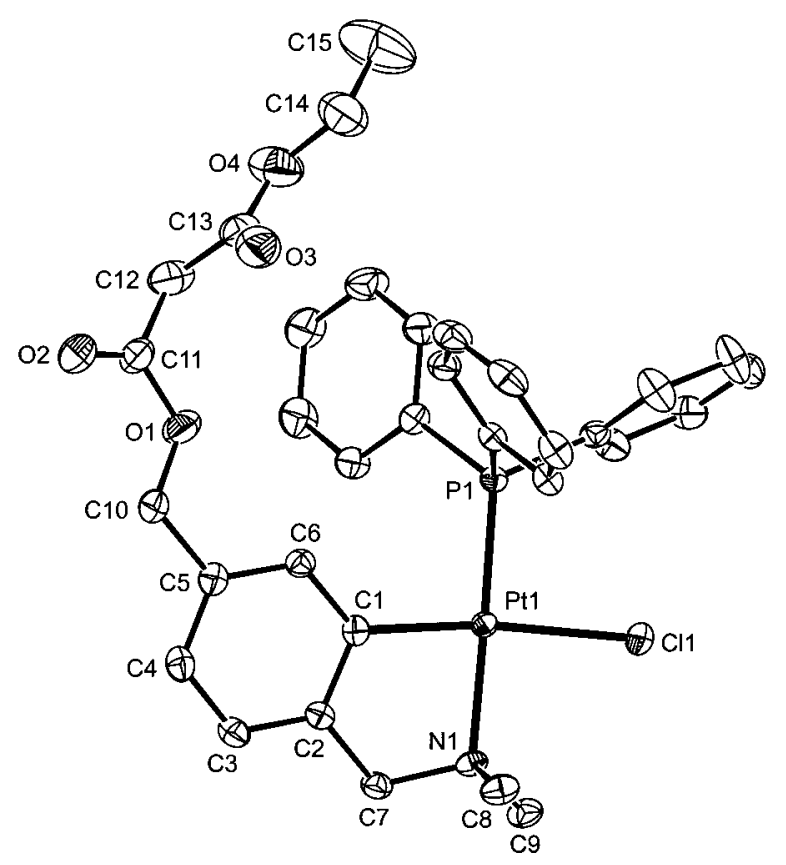

Figure 3. Displacement ellipsoid plot (50\% probability level) of the molecular structure of 10, with the adopted numbering scheme. The hydrogen atoms have been omitted for clarity.

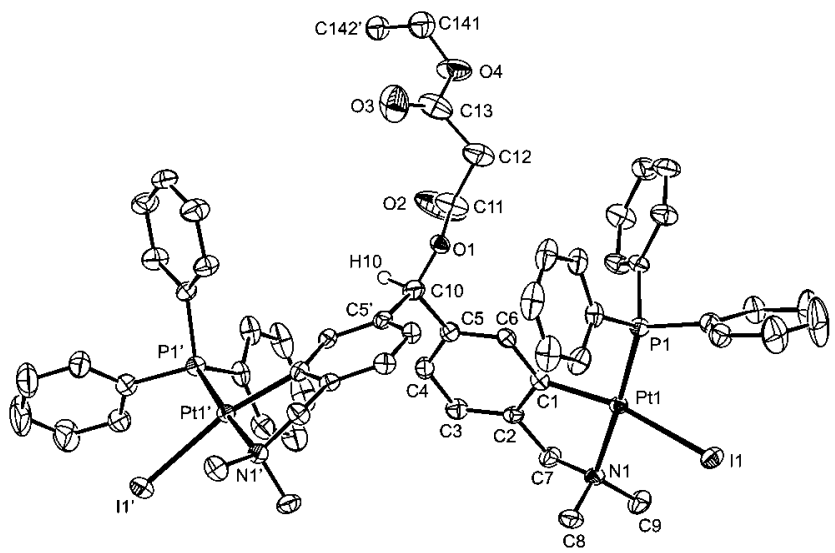

Figure 4. Displacement ellipsoid plot (50\% probability level) of the molecular structure of $\mathbf{1 3}$, with the adopted numbering scheme. All hydrogen atoms except $\mathrm{H}(10)$ have been omitted for clarity. The structure of $\mathbf{1 3}$ has an exact, crystallographic $\mathrm{C}_{2}$ symmetry, leading to rotational disorder at carbon C(10) and disorder of the ethyl malonyl chain and the hydrogen atom $\mathrm{H}(10)$ (both in $50 \%$ occupancy, only one disorder component is shown).

complexes $\mathbf{1 0}$ and $\mathbf{1 1})$, these complexes were converted into their corresponding iodide complexes $\mathbf{1 2}$ and $\mathbf{1 3}$ with $\mathrm{Nal}$ in acetone. The formation of these compounds was demonstrated by multinuclear NMR spectroscopy and elemental analysis. For $\mathbf{1 0}$ and $\mathbf{1 3}$, the X-ray crystal structure was also determined.

The molecular plots of the structures of $\mathbf{1 0}$ and $\mathbf{1 3}$ in the solid state are shown in Figures 3 and 4, respectively, while a selection of bond lengths and bond angles is presented in Table 3. The structure of $\mathbf{1 3}$ has an exact, crystall lographic $\mathrm{C}_{2}$ symmetry axis, leading to rotational disorder at carbon $\mathrm{C}(10)$ and disorder of the ethyl malonyl chain and the hydrogen atom $\mathrm{H}(10)$ (both in $50 \%$ occupancy). Only one disorder component is shown in Figure 4 (symmetry operation: $1-x, y, 0.5-z)$. The
Table 3. Selected Bond Distances $(\AA)$ and Angles (deg) of 10 and 13

\begin{tabular}{llll}
\hline \multicolumn{1}{c}{10} & & \multicolumn{1}{c}{13} \\
\hline $\mathrm{Pt}(1)-\mathrm{Cl}(1)$ & $2.3902(7)$ & $\mathrm{Pt}(1)-\mathrm{I}(1)$ & $2.6874(3)$ \\
$\mathrm{Pt}(1)-\mathrm{P}(1)$ & $2.2220(7)$ & $\mathrm{Pt}(1)-\mathrm{P}(1)$ & $2.2364(10)$ \\
$\mathrm{Pt}(1)-\mathrm{N}(1)$ & $2.138(2)$ & $\mathrm{Pt}(1)-\mathrm{N}(1)$ & $2.172(3)$ \\
$\mathrm{Pt}(1)-\mathrm{C}(1)$ & $2.008(3)$ & $\mathrm{Pt}(1)-\mathrm{C}(1)$ & $2.027(4)$ \\
$\mathrm{Cl}(1)-\mathrm{Pt}(1)-\mathrm{P}(1)$ & $91.37(2)$ & $\mathrm{I}(1)-\mathrm{Pt}(1)-\mathrm{P}(1)$ & $92.83(3)$ \\
$\mathrm{Cl}(1)-\mathrm{Pt}(1)-\mathrm{N}(1)$ & $90.02(6)$ & $\mathrm{I}(1)-\mathrm{Pt}(1)-\mathrm{N}(1)$ & $90.96(8)$ \\
$\mathrm{P}(1)-\mathrm{Pt}(1)-\mathrm{C}(1)$ & $96.47(8)$ & $\mathrm{P}(1)-\mathrm{Pt}(1)-\mathrm{C}(1)$ & $95.29(11)$ \\
$\mathrm{N}(1)-\mathrm{Pt}(1)-\mathrm{C}(1)$ & $81.95(10)$ & $\mathrm{N}(1)-\mathrm{Pt}(1)-\mathrm{C}(1)$ & $81.27(13)$ \\
$\mathrm{P}(1)-\mathrm{Pt}(1)-\mathrm{N}(1)$ & $178.01(6)$ & $\mathrm{P}(1)-\mathrm{Pt}(1)-\mathrm{N}(1)$ & $175.79(9)$ \\
$\mathrm{Cl}(1)-\mathrm{Pt}(1)-\mathrm{C}(1)$ & $168.50(7)$ & $\mathrm{I}(1)-\mathrm{Pt}(1)-\mathrm{C}(1)$ & $167.90(11)$ \\
interplanar angle & $9.51(14)$ & interplanar angle & $8.38(10)$ \\
between & & between & \\
$\mathrm{Pt}(1)-\mathrm{Cl}(1)-\mathrm{P}(1)$ and & & $\mathrm{Pt}(1)-\mathrm{I}(1)-\mathrm{P}(1)$ and \\
$\mathrm{Pt}(1)-\mathrm{N}(1)-\mathrm{C}(1)$ & & $\mathrm{Pt}(1)-\mathrm{N}(1)-\mathrm{C}(1)$ &
\end{tabular}

platinum centers in both $\mathbf{1 0}$ and $\mathbf{1 3}$ have a distorted square planar coordination geometry, reflected by the C,N-bite angles of $81.95(8)^{\circ}$ for 10 and $81.26(14)^{\circ}$ for 13, respectively. The ligand environment, i.e., the $\mathrm{PPh}_{3}$ ligands positioned trans to the $\mathrm{NMe}_{2}$ group and the chloride ligand trans to the aromatic ring, was not changed by the attachment of the malonyl group.

Derivatization of $\mathbf{C}_{60}$. The malonates $\mathbf{8}$ and $\mathbf{9}$ were attached to $\mathrm{C}_{60}$ via a Bingel cyclopropanation reaction in the presence of $\mathrm{I}_{2}$ and diazabicycl o[5.4.0]undec-7-ene (DBU) in toluene at room temperature (Scheme 5). The methanofullerenes $\mathbf{1 4}$ and $\mathbf{1 5}$ were characterized by NMR and mass spectroscopic analysis (MALDI-TOF). These methanofullerene $\mathrm{CH}, \mathrm{N}$ ligands were reacted with cis- $\mathrm{PtCl}_{2}(\mathrm{DMSO})_{2}$ and $\mathrm{NaOAC}$ in a mixture of $\mathrm{MeOH}$ and ortho-dichlorobenzene (ODCB, Scheme 5). Cycloplatination at the $\mathrm{C}, \mathrm{N}$-ligand took place for 14, affording the platinum(II)-DMSO complex 16, which was isolated in $57 \%$ yield. To our surprise, no bisplatinum complex starting from $\mathbf{1 5}$ was formed under the same conditions. Only starting material and insoluble products were isolated. However, direct attachment of the platinum complexes $\mathbf{1 2}$ and $\mathbf{1 3}$ to $\mathrm{C}_{60}$ in the presence of $\mathrm{I}_{2}$ and DBU did yield the new methanofullerene $\mathrm{C}, \mathrm{N}$ platinum complexes $\mathbf{1 7}$ and 18, respectively. Both $\mathbf{1 7}$ and 18 were isolated in reasonable yields $(60 \%$ and $59 \%$ based on reacted $\mathrm{C}_{60}$ ), which again demonstrates the stability of the $\mathrm{C}, \mathrm{N}$-platinum(II) building block. In the ${ }^{1} \mathrm{H}$ NMR spectrum of $\mathbf{1 7}$ and $\mathbf{1 8}$, the methylene resonance of the malonyl group of the starting material had disappeared, indicating a complete reaction, while the expected methanofullerene resonances in the 150-120 ppm region were observed by ${ }^{13} \mathrm{C}\left\{{ }^{1} \mathrm{H}\right\}$ NMR spectroscopy. The ${ }^{31} \mathrm{P}-{ }^{195} \mathrm{Pt}$ coupling constants in the ${ }^{31} \mathrm{P}\left\{{ }^{1} \mathrm{H}\right\}$ NMR spectrum of $4167 \mathrm{~Hz}$ (17) and $4174 \mathrm{~Hz}$ (18) showed that the platinum center remained in the same coordination environment during the Bingel reaction. Mass spectrometric analysis (MALDI-TOF) exhibited fragments at $\mathrm{m} / \mathrm{z} 1454.0$ (17) and 2170.1 (18), corresponding to the respective $[\mathrm{M}-\mathrm{I}]^{+}$fragments ions.

These results show that the chel ated platinum center is stable under the conditions of the cyclopropanation reaction. Apparently, the formation of the $\alpha$-halocarbanion and subsequent reaction with $\mathrm{C}_{60}$ is faster then the alternative $\mathrm{Pt}-\mathrm{C}(\mathrm{C}, \mathrm{N})$ bond cleavage. M oreover, we have found that the organometallic moiety of these complexes is al so stable to the chromatographic workup conditions traditionally used in fullerene chemistry. Grafting of a complete organometallic synthon to $\mathrm{C}_{60}$ is thus a useful route for the synthesis of methanofullerene $\mathrm{C}, \mathrm{N}$-platinum(II) complexes, especially in cases where 
Scheme 5. Derivatization of $\mathrm{C}_{60}{ }^{\mathrm{a}}$

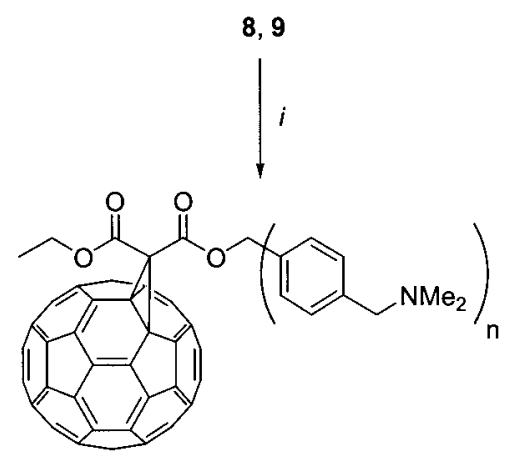

$14(n=1), 15 \quad(n=2)$

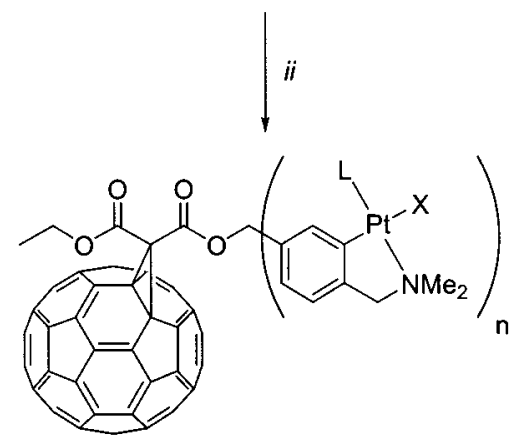

$16 \mathrm{~L}=\mathrm{DMSO}, \mathrm{X}=\mathrm{Cl}, \mathrm{n}=1$

$17 \mathrm{~L}=\mathrm{PPh}_{3}, X=\mathrm{I}, \mathrm{n}=1$

$18 \mathrm{~L}=\mathrm{PPh}_{3}, X=1, \mathrm{n}=2$

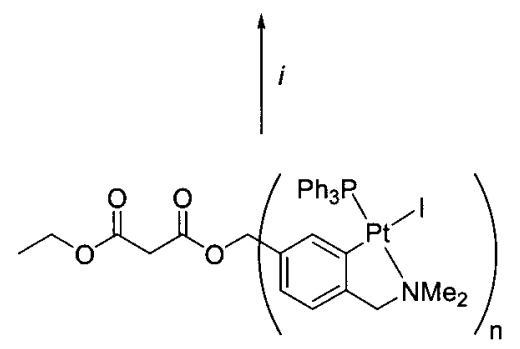

$12(n=1), 13 \quad(n=2)$

a Reagents and Conditions: (i) $\mathrm{C}_{60}, \mathrm{I}_{2}, \mathrm{DBU}$, toluene, $r \mathrm{r}, 24$ h; (ii) cis-PtCl (DMSO) $_{2}, \mathrm{NaOAC}, \mathrm{MeOH} / \mathrm{DCB}(1: 2 \mathrm{v} / \mathrm{v} \%), 65^{\circ} \mathrm{C}$.

the methanofullerene ligand cannot be directly metalated. In summary, the presented C,N-platinum compounds have been shown to be versatile and stable complexes, suitable in dendrimer and fullerene chemistry. These complexes are also promising building blocks for other areas of macromolecular chemistry, e.g., for materials based on the use of metal $d^{8}$ complexes. ${ }^{2}$

\section{Experimental Section}

General Comments. All experiments were conducted under a dry dinitrogen atmosphere using standard Schlenk techniques. Solvents were dried over appropriate materials and distilled prior to use. ${ }^{1} \mathrm{H}$ and ${ }^{13} \mathrm{C}\left\{{ }^{1} \mathrm{H}\right\}$ NMR spectra were recorded at $298 \mathrm{~K}$ (unless stated otherwise) on a Varian Inova $300 \mathrm{MHz}$ spectrometer, ${ }^{31} \mathrm{P}\left\{{ }^{1} \mathrm{H}\right\} \mathrm{NMR}$ spectra were recorded on a Varian Mercury $200 \mathrm{MHz}$ NMR spectrometer. All NMR chemical shifts are in ppm referenced to residual solvent signals $\left({ }^{1} \mathrm{H}\right.$ and $\left.{ }^{13} \mathrm{C}\left\{{ }^{1} \mathrm{H}\right\}\right)$ or to $\mathrm{H}_{3} \mathrm{PO}_{4}\left({ }^{31} \mathrm{P}\left\{{ }^{1} \mathrm{H}\right\}\right)$. The starting materials cis- $\mathrm{PtCl}_{2}\left(\mathrm{DMSO}_{2},{ }^{15}\right.$ 4-[(dimethylamino)methyl]bromobenzene, ${ }^{6} 4-\left[\left(\right.\right.$ dimethylamino)methyl ]benzaldehyde, ${ }^{16}$ and the F réchet dendron [G1]-COCl${ }^{14}$ were prepared according to literature procedures. $\mathrm{C}_{60}$ (Hoechst, Gold Grade) was used as received. The MALDI-TOF mass spectra were acquired using a Voyager-DE BioSpectrometry Workstation mass spectrometer (PerSeptive Biosystems I nc., Framingham, MA). Sample solutions with an approximate concentration of $1 \mathrm{~g} \mathrm{~L}^{-1}$ in $\mathrm{CH}_{2-}$ $\mathrm{Cl}_{2}$ were prepared. The matrix was 9-nitroanthracene (9-NA) with an approximate concentration of $40-50 \mathrm{~g} \mathrm{~L}^{-1}$. An $0.2 \mu \mathrm{L}$ aliquot of the sample solution and $0.2 \mu \mathrm{L}$ of the matrix solution were combined on a golden MALDI target and analyzed after evaporation of the solvent. Elemental analyses were performed by Dornis und Kol be, Mikroanalytisches Laboratorium (Mülheim, Germany).

4-[(Dimethylamino)methyl]benzyl Alcohol (1). To a suspension of $\mathrm{LiAlH}_{4}(3.27 \mathrm{~g}, 86.2 \mathrm{mmol})$ in $\mathrm{Et}_{2} \mathrm{O}(75 \mathrm{~mL})$ at 0 ${ }^{\circ} \mathrm{C}$ was added a solution of 4-[(dimethylamino)methyl]benzaldehyde $(7.03 \mathrm{~g}, 43.1 \mathrm{mmol})$ in $\mathrm{Et}_{2} \mathrm{O}(40 \mathrm{~mL})$. The reaction mixture was stirred for $20 \mathrm{~h}$, car efully quenched with $10 \mathrm{~mL}$ of a saturated aqueous solution of $\mathrm{NaCl}$, and filtered. The organic fraction was separated, dried over $\mathrm{MgSO}_{4}$, filtered, and evaporated in vacuo to yield $\mathbf{1}$ as a colorless oil (4.97 g, 77\%). ${ }^{1} \mathrm{H} N M R\left(\mathrm{CDCl}_{3}\right): \delta 7.31(\mathrm{~d}, 2 \mathrm{H}, 3 \mathrm{j}=8.7 \mathrm{~Hz}, \mathrm{Ar}-\mathrm{H}), 7.26(\mathrm{~d}$, $\left.2 \mathrm{H},{ }^{3} \mathrm{~J}=8.4 \mathrm{~Hz}, \mathrm{Ar}-\mathrm{H}\right), 4.65\left(\mathrm{~s}, 2 \mathrm{H}, \mathrm{CH}_{2} \mathrm{OH}\right), 3.40(\mathrm{~s}, 2 \mathrm{H}$, $\left.\mathrm{CH}_{2} \mathrm{~N}\right), 3.06(\mathrm{~s}, 1 \mathrm{H}, \mathrm{OH}), 2.20\left(\mathrm{~s}, 6 \mathrm{H}, \mathrm{NCH}_{3}\right) .{ }^{13} \mathrm{C}\left\{{ }^{1} \mathrm{H}\right\} \mathrm{NMR}$ $\left(\mathrm{CDCl}_{3}\right): \delta 140.1,137.6,129.3,126.9$ (aryl-C), $64.9(\mathrm{COH}), 64.0$ $\left(\mathrm{CH}_{2} \mathrm{~N}\right)$, $45.1\left(\mathrm{NCH}_{3}\right)$. Anal. Calcd for $\mathrm{C}_{10} \mathrm{H}_{15} \mathrm{NO}$ : C 72.69, $\mathrm{H}$ 9.15, N 8.48. Found: C 72.56, H 9.21, N 8.44.

3-[Chloro(DMSO)platino]-4-[(dimethylamino)methyl]benzyl Alcohol (2). To a solution of 1 (1.06 g, $6.42 \mathrm{mmol})$ in $\mathrm{MeOH}(40 \mathrm{~mL})$ was added cis- $\mathrm{PtCl}_{2}(\mathrm{DMSO})_{2}(2.77 \mathrm{~g}, 6.55$ $\mathrm{mmol})$ and $\mathrm{NaOAc}(0.54 \mathrm{~g}, 6.6 \mathrm{mmol})$. The reaction mixture was stirred at $65^{\circ} \mathrm{C}$ for $3 \mathrm{~h}$, whereupon a white solid separated from the solution. The product was collected by decantation, washed with $\mathrm{MeOH}(50 \mathrm{~mL})$ and $\mathrm{Et}_{2} \mathrm{O}(2 \times 50 \mathrm{~mL})$, and dried in vacuo, yielding 2 as a white solid $(2.00 \mathrm{~g}, 66 \%)$. ${ }^{1} \mathrm{H}$ NMR $\left(\mathrm{CDCl}_{3}\right): \delta 7.92\left(\mathrm{~s}, 3 \mathrm{~J}(\mathrm{Pt}-\mathrm{H})=47.4 \mathrm{~Hz}, 1 \mathrm{H}, \mathrm{Ar}-\mathrm{H}_{\text {ortho }}\right), 7.11$ $\left(\mathrm{d},{ }^{2} \mathrm{~J}=7.80 \mathrm{~Hz}, 1 \mathrm{H}, \mathrm{Ar}-\mathrm{H}\right), 7.06\left(\mathrm{~d},{ }^{2} \mathrm{~J}=7.80 \mathrm{~Hz}, 1 \mathrm{H}, \mathrm{Ar}-\mathrm{H}\right)$, $4.64\left(\mathrm{~d},{ }^{2} \mathrm{~J}=6.0 \mathrm{~Hz}, 2 \mathrm{H}, \mathrm{CH}_{2} \mathrm{O}\right), 3.98(\mathrm{~s}, 3 \mathrm{~J}(\mathrm{Pt}-\mathrm{H})=39.9 \mathrm{~Hz}$, $\left.2 \mathrm{H}, \mathrm{CH}_{2} \mathrm{~N}\right), 3.54\left(\mathrm{~s}, 3 \mathrm{~J}(\mathrm{Pt}-\mathrm{H})=24.9 \mathrm{~Hz}, 6 \mathrm{H}, \mathrm{SCH}_{3}\right), 2.90(\mathrm{~s}$, 3) $\left.(\mathrm{Pt}-\mathrm{H})=33.9 \mathrm{~Hz}, 6 \mathrm{H}, \mathrm{NCH}_{3}\right), 1.70\left(\mathrm{t},{ }^{2} \mathrm{~J}=6.0 \mathrm{~Hz}, 1 \mathrm{H}, \mathrm{OH}\right)$. ${ }^{13} \mathrm{C}\left\{{ }^{1} \mathrm{H}\right\} \mathrm{NMR}\left(\mathrm{CDCl}_{3}\right): \delta 145.5,138.7\left({ }^{3} \mathrm{~J}(\mathrm{Pt}-\mathrm{C})=53.3 \mathrm{~Hz}\right)$, $136.2\left(\mathrm{~J}(\mathrm{Pt}-\mathrm{C})=1061.3 \mathrm{~Hz}, \mathrm{Ar}-\mathrm{C}_{\mathrm{ipso}}\right), 132.9$ ( $^{2} \mathrm{~J}(\mathrm{Pt}-\mathrm{C})=54.0$ $\mathrm{Hz}), 123.9,121.8(3 \mathrm{~J}(\mathrm{Pt}-\mathrm{C})=36.4 \mathrm{~Hz}), 74.7(2 \mathrm{j}(\mathrm{Pt}-\mathrm{C})=51.5$ $\left.\mathrm{Hz}, \mathrm{CH}_{2} \mathrm{~N}\right), 65.8(\mathrm{COH}), 52.2\left(\mathrm{NCH}_{3}\right), 46,8$ ( ${ }^{2} \mathrm{~J}(\mathrm{Pt}-\mathrm{C})=63.1$ $\mathrm{Hz}, \mathrm{SCH}_{3}$ ). Anal. Calcd for $\mathrm{C}_{12} \mathrm{H}_{20} \mathrm{CINO}_{2} \mathrm{PtS}$ : $\mathrm{C} 30.48, \mathrm{H} 4.26$, N 2.96. Found: C 30.34, H 4.21, N 2.85.

3-[Chloro(triphenylphosphine)platino]-4-[(dimethylamino)methyl]benzyl Alcohol (3). To a suspension of $\mathbf{2}$ (1.01 g, $2.14 \mathrm{mmol})$ in $\mathrm{CH}_{2} \mathrm{Cl}_{2}(40 \mathrm{~mL})$ was added $\mathrm{PPh}_{3}(0.59$ $\mathrm{g}, 2.2 \mathrm{mmol}$ ). The reaction mixture was stirred for $2 \mathrm{~h}$, filtered through Celite, and evaporated in vacuo. The crude product was washed with $\mathrm{Et}_{2} \mathrm{O}(2 \times 50 \mathrm{~mL})$ and dried in vacuo, yielding 3 as a white solid $(0.90 \mathrm{~g}, 64 \%)$. ${ }^{1} \mathrm{H} \mathrm{NMR}\left(\mathrm{CDCl}_{3}\right): \delta 7.79-$ $7.72(\mathrm{~m}, 6 \mathrm{H}, \mathrm{PAr}-\mathrm{H}), 7.44-7.32(\mathrm{~m}, 9 \mathrm{H}, \mathrm{PAr}-\mathrm{H}), 7.04\left(\mathrm{~d},{ }^{3} \mathrm{~J}=\right.$ $7.2 \mathrm{~Hz}, 1 \mathrm{H}, \mathrm{Ar}-\mathrm{H}), 6.82(\mathrm{dd}, 3 \mathrm{~J}=7.7 \mathrm{~Hz}, 4 \mathrm{~J}=1.8 \mathrm{~Hz}, 1 \mathrm{H}$, Ar-H), $6.34(\mathrm{dd}, 3 \mathrm{~J}(\mathrm{Pt}-\mathrm{H})=56.7 \mathrm{~Hz}$, $4(\mathrm{P}-\mathrm{H})=2.6 \mathrm{~Hz}, 4 \mathrm{~J}=$ $\left.1.8 \mathrm{~Hz}, 1 \mathrm{H}, \mathrm{Ar}-\mathrm{H}_{\text {ortho }}\right), 4.06(\mathrm{~d}, 3 \mathrm{~J}(\mathrm{Pt}-\mathrm{H})=29.7 \mathrm{~Hz}, 3 \mathrm{~J}(\mathrm{P}-\mathrm{H})$ $\left.=3.0 \mathrm{~Hz}, 2 \mathrm{H}, \mathrm{CH}_{2} \mathrm{~N}\right), 3.96\left(\mathrm{~d},{ }^{3} \mathrm{j}=6.0 \mathrm{~Hz}, 2 \mathrm{H}, \mathrm{CH}_{2} \mathrm{O}\right), 2.97(\mathrm{~d}$, 3) $(\mathrm{Pt}-\mathrm{H})=24.3 \mathrm{~Hz}$, 3 $\left.(\mathrm{P}-\mathrm{H})=3.3 \mathrm{~Hz}, 6 \mathrm{H}, \mathrm{NCH}_{3}\right), 0.68$ (t, 3) $=6.0 \mathrm{~Hz}, 1 \mathrm{H}, \mathrm{OH}) .{ }^{13} \mathrm{C}\left\{{ }^{1} \mathrm{H}\right\} \mathrm{NMR}\left(\mathrm{CDCl}_{3}\right): \delta 146.91(\mathrm{Ar}-\mathrm{C})$, $138.04\left(\mathrm{~d}\right.$, J $\left.~(P-C)=6.7 \mathrm{~Hz}, \mathrm{PAr}-\mathrm{C}_{\mathrm{ipso}}\right), 137.57(\mathrm{~d}$, 2 $\mathrm{J}(\mathrm{P}-\mathrm{C})=$ $2.4 \mathrm{~Hz}, \mathrm{Ar}-\mathrm{C}), 135.29(\mathrm{~d}, \mathrm{2})(\mathrm{P}-\mathrm{C})=10.4 \mathrm{~Hz}$, 3 $(\mathrm{Pt}-\mathrm{C})=39.5$ $\mathrm{Hz}, \mathrm{PAr}-\mathrm{C}_{\text {ortho}}$ ), 130.77 (Ar-C), 130.55 (d, 4J $(\mathrm{P}-\mathrm{C})=2.4 \mathrm{~Hz}$, Ar- $\left.\mathrm{C}_{\text {para }}\right), 129.95$ (3) (Pt-C) $\left.=36.3 \mathrm{~Hz}, \mathrm{Ar}-\mathrm{C}\right), 127.82$ (d, 3) (PC) $\left.=10.9 \mathrm{~Hz}, \mathrm{PAr}-\mathrm{C}_{\text {meta }}\right), 121.93(\mathrm{Ar}-\mathrm{C}), 121.66(\mathrm{~J}(\mathrm{Pt}-\mathrm{C})=$ 35.3 Hz, Ar-C), 74.0 ( $\left.{ }^{2} \mathrm{~J}(\mathrm{Pt}-\mathrm{C})=50.3 \mathrm{~Hz}, \mathrm{CH}_{2} \mathrm{~N}\right), 65.3(\mathrm{COH})$, $50.7\left(\mathrm{NCH}_{3}\right) .{ }^{31} \mathrm{P}\left\{{ }^{1} \mathrm{H}\right\} \mathrm{NMR}\left(\mathrm{CDCl}_{3}\right): \delta 20.7\left({ }^{1} \mathrm{~J}(\mathrm{Pt}-\mathrm{P})=4261\right.$ $\mathrm{Hz}$ ). Anal. Calcd for $\mathrm{C}_{28} \mathrm{H}_{29} \mathrm{CINOPPt}$ : C 51.18, H 4.45, N 2.13. Found: C 51.18, H 4.55, N 2.04.

(15) Price, J . H.; Williamson, A. N.; Schramm, R. F.; Wayland, B. B. Inorg. Chem. 1972, 11, 1280.

(16) Meijer, M. D.; de Wolf, E.; Lutz, M.; Spek, A. L.; van Klink, G. P. M.; van Koten, G. Organometallics 2001, 20, 4198. 
Bis(4-[(dimethylamino)methyl]phenyl)methyl Alcohol (4). To a solution of 4-[(dimethylamino)methyl ]bromobenzene $(5.80 \mathrm{~g}, 27.1 \mathrm{mmol})$ in $\mathrm{Et}_{2} \mathrm{O}(30 \mathrm{~mL})$ at $-78{ }^{\circ} \mathrm{C}$ was added 37 $\mathrm{mL}$ of a $1.5 \mathrm{M}$ solution of t-BuLi in pentane $(55 \mathrm{mmol})$. The reaction mixture was stirred at $-78{ }^{\circ} \mathrm{C}$ for $1 \mathrm{~h}$, and a solution of 4-[(dimethylamino)methyl ]benzal dehyde $(4.43 \mathrm{~g}, 27.1 \mathrm{mmol})$ in $\mathrm{Et}_{2} \mathrm{O}(25 \mathrm{~mL})$ was added in one portion. The reaction mixture was allowed to warm to room temperature over $2 \mathrm{~h}$ and was quenched with water $(50 \mathrm{~mL})$. The organic layer was separated, and the water layer extracted with $\mathrm{Et}_{2} \mathrm{O}(2 \times 100$ $\mathrm{mL}$ ). The organic fractions were dried on $\mathrm{MgSO}_{4}$, filtered, and evaporated in vacuo. The crude product was purified using Kugel rohr distillation giving $\mathbf{4}$ as a yellow oil, which solidified upon standing $(6.23 \mathrm{~g}, 77 \%)$. ${ }^{1} \mathrm{H}$ NMR $\left(\mathrm{CDCl}_{3}\right): \delta 7.29(\mathrm{~d}, 4 \mathrm{H}$, 3) $=8.4 \mathrm{~Hz}, \mathrm{Ar}-\mathrm{H}), 7.20(\mathrm{~d}, 4 \mathrm{H}$, 3) $=8.1 \mathrm{~Hz}, \mathrm{Ar}-\mathrm{H}), 5.71(\mathrm{~s}$, $1 \mathrm{H}, \mathrm{CHO}), 5.08(\mathrm{~s}, 1 \mathrm{H}, \mathrm{OH}), 3.34\left(\mathrm{~s}, 4 \mathrm{H}, \mathrm{CH}_{2} \mathrm{~N}\right), 2.13(\mathrm{~s}, 12 \mathrm{H}$, $\left.\mathrm{NCH}_{3}\right) .{ }^{13} \mathrm{C}\left\{{ }^{1} \mathrm{H}\right\} \mathrm{NMR}\left(\mathrm{CDCl}_{3}\right): \delta 143.5,137.0,129.2,126.4$ $(\mathrm{Ar}-\mathrm{C}), 75.4(\mathrm{COH}), 63.8\left(\mathrm{CH}_{2} \mathrm{~N}\right), 45.0\left(\mathrm{NCH}_{3}\right)$. Anal. Calcd for $\mathrm{C}_{19} \mathrm{H}_{26} \mathrm{~N}_{2} \mathrm{O}$ : C 76.47, H 8.78, N 9.39. Found: C 76.29, H 8.90, N 9.26 .

Bis(3-[chloro (DMSO) platino]-4-[(dimethylamino)methyl]phenyl)methyl Alcohol (5). Complex $\mathbf{5}$ was synthesized using the same method as that described for 2 starting from $4(0.35 \mathrm{~g}, 1.2 \mathrm{mmol})$, cis- $\mathrm{PtCl}_{2}(\mathrm{DMSO})_{2}(1.01 \mathrm{~g}$, $2.39 \mathrm{mmol})$, and $\mathrm{NaOAc}(0.21 \mathrm{~g}, 2.6 \mathrm{mmol})$. Complex 5 was isolated as a white solid $(0.91 \mathrm{~g}, 84 \%) .{ }^{1} \mathrm{H} \mathrm{NMR}\left(\mathrm{CDCl}_{3}\right): \delta$ $8.03\left(\mathrm{~d},{ }^{3} \mathrm{~J}(\mathrm{Pt}-\mathrm{H})=48.0 \mathrm{~Hz}, 4 \mathrm{~J}=1.2 \mathrm{~Hz}, 2 \mathrm{H}, \mathrm{Ar}-\mathrm{H}_{\text {ortho }}\right), 7.13$ $\left(\mathrm{dd},{ }^{3} \mathrm{~J}=7.5 \mathrm{~Hz},{ }^{4} \mathrm{~J}=1.2 \mathrm{~Hz}, 2 \mathrm{H}, \mathrm{Ar}-\mathrm{H}\right), 6.99(\mathrm{~d}, 3)=8.1 \mathrm{~Hz}$, $2 \mathrm{H}, \mathrm{Ar}-\mathrm{H}$ ), $5.77\left(\mathrm{~d},{ }^{3} \mathrm{~J}=3.0 \mathrm{~Hz}, 1 \mathrm{H}, \mathrm{CHO}\right), 3.94 / 3.91$ (dd, $\mathrm{AB}$, $\left.\left.{ }^{2} \mathrm{~J}=13.7 \mathrm{~Hz},{ }^{3} \mathrm{Pt}-\mathrm{P}\right)=36.9 \mathrm{~Hz}, 4 \mathrm{H}, \mathrm{CH}_{2} \mathrm{~N}\right), 3.50\left(\mathrm{~s},{ }^{3} \mathrm{~J}(\mathrm{Pt}-\right.$ $\left.\mathrm{H})=21.6 \mathrm{~Hz}, 12 \mathrm{H}, \mathrm{SCH}_{3}\right), 2.89,2.88(2 \times \mathrm{s}, 3 \mathrm{~J}(\mathrm{Pt}-\mathrm{H})=31.5$ $\left.\mathrm{Hz}, 12 \mathrm{H}, \mathrm{NCH}_{3}\right), 2.29\left(\mathrm{~d},{ }^{3} \mathrm{~J}=3.3 \mathrm{~Hz}, 1 \mathrm{H}, \mathrm{HOCH}\right) .{ }^{13} \mathrm{C}\left\{{ }^{1} \mathrm{H}\right\}$ NMR $\left(\mathrm{CDCl}_{3}\right): \delta 145.0(4 \mathrm{~J}(\mathrm{Pt}-\mathrm{C})=18.2 \mathrm{~Hz}), 142.5,136.3$, 132.5 ( $\mathrm{(J}(\mathrm{Pt}-\mathrm{C})=55.2 \mathrm{~Hz}, \mathrm{Ar}-\mathrm{C}), 123.3(\mathrm{Ar}-\mathrm{C}), 121.9$ (3) (Pt$\mathrm{C})=37.7 \mathrm{~Hz}, \mathrm{Ar}-\mathrm{C}), 77.1(\mathrm{COH}), 74.9(\mathrm{~J}(\mathrm{Pt}-\mathrm{C})=53.0 \mathrm{~Hz}$, $\left.\mathrm{CH}_{2} \mathrm{~N}\right), 52.43,52.38\left(\mathrm{NCH}_{3}\right), 47.0$ ( $\left(\mathrm{J}(\mathrm{Pt}-\mathrm{C})=64.9 \mathrm{~Hz}, \mathrm{SCH}_{3}\right)$. Anal. Calcd for $\mathrm{C}_{23} \mathrm{H}_{36} \mathrm{Cl}_{2} \mathrm{~N}_{2} \mathrm{O}_{3} \mathrm{Pt}_{2} \mathrm{~S}_{2}$ : C 53.79, $\mathrm{H}$ 7.05, N 7.84. Found: C 53.88, $\mathrm{H}$ 7.11, N 7.86.

Bis(3-[chloro(triphenylphosphine)platino]-4-[(dimethylamino)methyl]phenyl)methyl Alcohol (6). Complex 6 was synthesized using the same method as that described for 3 starting from $5(0.47 \mathrm{~g}, 0.51 \mathrm{mmol})$ and $\mathrm{PPh}_{3}(0.27 \mathrm{~g}, 1.0$ $\mathrm{mmol})$, yielding 6 as a white solid $(0.52 \mathrm{~g}, 80 \%)$. ${ }^{1} \mathrm{H}$ NMR $\left(\mathrm{CDCl}_{3}\right): \delta 7.75-7.67(\mathrm{~m}, 12 \mathrm{H}, \mathrm{PAr}-\mathrm{H}), 7.42-7.29(\mathrm{~m}, 18 \mathrm{H}$, PAr-H), $6.70(\mathrm{~d}, 3 \mathrm{~J}=7.5 \mathrm{~Hz}, 2 \mathrm{H}, \mathrm{Ar}-\mathrm{H}), 6.43(\mathrm{dd}, 3 \mathrm{~J}(\mathrm{Pt}-\mathrm{H})=$ $57.3 \mathrm{~Hz}$, 顶 $\left.(\mathrm{P}-\mathrm{H})=2.9 \mathrm{~Hz}, 4 \mathrm{~J}=1.3 \mathrm{~Hz}, 2 \mathrm{H}, \mathrm{Ar}-\mathrm{H}_{\text {ortho }}\right), 5.90$ $(\mathrm{dd}, 3 \mathrm{~J}=7.7 \mathrm{~Hz}, 4 \mathrm{~J}=1.3 \mathrm{~Hz}, 2 \mathrm{H}, \mathrm{Ar}-\mathrm{H}), 4.35(\mathrm{~d}, 3 \mathrm{~J}=3.6 \mathrm{~Hz}$, $1 \mathrm{H}, \mathrm{CHO}), 4.02 / 3.98\left(\mathrm{dd}, \mathrm{AB},{ }^{2} \mathrm{~J}=13.7 \mathrm{~Hz},{ }^{4} \mathrm{~J}(\mathrm{P}-\mathrm{H})=3.0 \mathrm{~Hz}\right.$, 3) $\left.(\mathrm{Pt}-\mathrm{H})=30.5 \mathrm{~Hz}, 4 \mathrm{H}, \mathrm{CH}_{2} \mathrm{~N}\right), 2.97,2.95(2 \times \mathrm{d}$, 邻 $(\mathrm{P}-\mathrm{H})=$ $2.7 \mathrm{~Hz}$, 3j $(\mathrm{Pt}-\mathrm{H})$ not resolved, $\left.12 \mathrm{H}, \mathrm{NCH}_{3}\right), 0.52(\mathrm{~d}$, 3 $3=3.6$ $\mathrm{Hz}, 1 \mathrm{H}, \mathrm{OH}) .{ }^{13} \mathrm{C}\left\{{ }^{1} \mathrm{H}\right\} \mathrm{NMR}\left(\mathrm{CDCl}_{3}\right): \delta 146.0(\mathrm{Ar}-\mathrm{C}), 140.68$ $\left(\mathrm{d},{ }^{2} \mathrm{~J}(\mathrm{P}-\mathrm{C})=2.3 \mathrm{~Hz}, \mathrm{Ar}-\mathrm{C}\right), 136.84\left(\mathrm{~d},{ }^{1} \mathrm{~J}(\mathrm{P}-\mathrm{C})=6.8 \mathrm{~Hz}, \mathrm{PAr}-\right.$ $\left.\mathrm{C}_{\text {ipso }}\right), 135.18$ (d, 2J $\left.(\mathrm{P}-\mathrm{C})=11.0 \mathrm{~Hz}, \mathrm{PAr}-\mathrm{C}_{\text {ortho }}\right), 130.89$ (ArC), $130.41\left(\mathrm{~d}, 4 \mathrm{~J}(\mathrm{P}-\mathrm{C})=2.4 \mathrm{~Hz}, \mathrm{PAr}-\mathrm{C}_{\mathrm{para}}\right), 130.08$ (Ar-C), $127.78\left(\mathrm{~d},{ }^{3} \mathrm{~J}(\mathrm{P}-\mathrm{C})=11.0 \mathrm{~Hz}, \mathrm{PAr}-\mathrm{C}_{\text {meta }}\right), 121.15(\mathrm{Ar}-\mathrm{C}), 120.95$ (Ar-C), $76.2(\mathrm{COH}), 74.1$ ( $\left.\mathrm{J}(\mathrm{Pt}-\mathrm{C})=52.5 \mathrm{~Hz}, \mathrm{CH}_{2} \mathrm{~N}\right), 50.9$, $50.7\left(\mathrm{NCH}_{3}\right) .{ }^{31} \mathrm{P}\left\{{ }^{1} \mathrm{H}\right\} \mathrm{NMR}\left(\mathrm{CDCl}_{3}\right): \delta 21.1$ ( ${ }^{1} \mathrm{~J}(\mathrm{Pt}-\mathrm{P})=4281$ $\mathrm{Hz}$ ). Anal. Calcd for $\mathrm{C}_{55} \mathrm{H}_{54} \mathrm{Cl}_{2} \mathrm{~N}_{2} \mathrm{OP}_{2} \mathrm{Pt}_{2}$ : C 51.53, $\mathrm{H} 4.25, \mathrm{~N}$ 2.19. Found: C 51.34, H 4.30, N 2.20.

\{3-[Chloro(triphenylposphine)platino]-4-[(dimethylamino)methyl]benzyl\}-[G1]-carboxylate (7). To a solution of $4(0.67 \mathrm{~g}, 1.0 \mathrm{mmol})$ in $\mathrm{CH}_{2} \mathrm{Cl}_{2}(25 \mathrm{~mL})$ was added $\mathrm{NEt}_{3}$ $(0.20 \mathrm{~mL}, 1.4 \mathrm{mmol})$. A solution of the Fréchet dendron [G1]$\mathrm{C}(\mathrm{O}) \mathrm{Cl}(0.36 \mathrm{~g}, 1.0 \mathrm{mmol})$ in $\mathrm{CH}_{2} \mathrm{Cl}_{2}(25 \mathrm{~mL})$ was added dropwisely, and the solution was stirred at room temperature for 3 days. The reaction mixture was washed with an aqeous saturated $\mathrm{NaCl}$ solution ( $50 \mathrm{~mL}$ ), dried over $\mathrm{MgSO}_{4}$, filtered, and evaporated in vacuo. The remaining solid was washed with $\mathrm{Et}_{2} \mathrm{O}(2 \times 15 \mathrm{~mL})$ and dried in vacuo, yielding 7 as a white solid $(0.65 \mathrm{~g}, 65 \%)$. ${ }^{1} \mathrm{H}$ NMR $\left(\mathrm{CDCl}_{3}\right): \delta 7.76-7.68(\mathrm{~m}, 6 \mathrm{H}, \mathrm{Ar}-$ $\mathrm{H}), 7.46-7.27(\mathrm{~m}, 19 \mathrm{H}, \mathrm{Ar}-\mathrm{H}), 7.13(\mathrm{~d}, 4 \mathrm{~J}=2.5 \mathrm{~Hz}, 2 \mathrm{H}, \mathrm{Ar}-$
$\mathrm{H}), 7.04(\mathrm{~d}, 3 \mathrm{~J}=7.8 \mathrm{~Hz}, 1 \mathrm{H}, \mathrm{Ar}-\mathrm{H}), 6.92(\mathrm{dd}, 3 \mathrm{~J}=7.4 \mathrm{~Hz}, 4 \mathrm{~J}$ $=1.6 \mathrm{~Hz}, 1 \mathrm{H}, \mathrm{Ar}-\mathrm{H}), 6.80(\mathrm{t}, 4 \mathrm{~J}=2.4 \mathrm{~Hz}, 1 \mathrm{H}, \mathrm{Ar}-\mathrm{H}), 6.43$ $(\mathrm{dd}$, 3 $(\mathrm{Pt}-\mathrm{H})=52.8 \mathrm{~Hz}$, 4 $(\mathrm{P}-\mathrm{H})=3.0 \mathrm{~Hz}$, 4 $=1.5 \mathrm{~Hz}, 2 \mathrm{H}$, Ar- $\left.\mathrm{H}_{\text {ortho }}\right), 5.06\left(\mathrm{~s}, 4 \mathrm{H}, \mathrm{CH}_{2} \mathrm{O}\right), 4.62\left(\mathrm{~s}, 2 \mathrm{H}, \mathrm{CH}_{2} \mathrm{O}\right), 4.07$ (d, 3) $(\mathrm{Pt}-\mathrm{H})=27.1 \mathrm{~Hz}$, 3 $\left.(\mathrm{P}-\mathrm{H})=3.0 \mathrm{~Hz}, 2 \mathrm{H}, \mathrm{CH}_{2} \mathrm{~N}\right), 2.99(\mathrm{~d}$, 3) $(\mathrm{Pt}-\mathrm{H})=23.4 \mathrm{~Hz}$, 3j $\left.(\mathrm{P}-\mathrm{H})=2.8 \mathrm{~Hz}, 6 \mathrm{H}, \mathrm{NCH}_{3}\right) .{ }^{13} \mathrm{C}\left\{{ }^{1} \mathrm{H}\right\}$ NMR $\left(\mathrm{CDCl}_{3}\right): \delta 165.74,159.60,147.53(\mathrm{Ar}-\mathrm{C}), 137.63$ ( ${ }^{1} \mathrm{~J}(\mathrm{P}-$ C) $\left.=7.1 \mathrm{~Hz}, \mathrm{PAr}-\mathrm{C}_{\mathrm{ipso}}\right), 137.48\left({ }^{\mathrm{J}}(\mathrm{Pt}-\mathrm{C})=86.7 \mathrm{~Hz}, \mathrm{Ar}-\mathrm{C}\right)$ 136.40, 135.15 (d, 2J $\left.(P-C)=10.9 \mathrm{~Hz}, \mathrm{PAr}-\mathrm{C}_{\text {ortho }}\right), 132.22$ (2) $(\mathrm{Pt}-\mathrm{C})=72.3 \mathrm{~Hz}, \mathrm{Ar}-\mathrm{C}), 132.11(\mathrm{Ar}-\mathrm{C}), 130.54$ (PAr-C para ), 129.77 (4) $(\mathrm{Pt}-\mathrm{C})=31.5 \mathrm{~Hz}, \mathrm{Ar}-\mathrm{C}), 128.63,128.16,128.03$, $127.75\left(\mathrm{~d},{ }^{3} \mathrm{f}(\mathrm{P}-\mathrm{C})=10.9 \mathrm{~Hz}, \mathrm{PAr}-\mathrm{C}_{\text {meta }}\right), 127.58,123.13(\mathrm{Ar}-$ C), 121.57 ( 4 ( $\mathrm{Pt}-\mathrm{C})=32.2 \mathrm{~Hz}, \mathrm{Ar}-\mathrm{C}), 108.73,106.43,74.1$ (2) $\left.(\mathrm{Pt}-\mathrm{C})=51.0 \mathrm{~Hz}, \mathrm{CH}_{2} \mathrm{~N}\right), 70.3\left(\mathrm{OCH}_{2}\right), 67.0\left(\mathrm{OCH}_{2}\right), 50.8$ $\left(\mathrm{NCH}_{3}\right) .{ }^{31} \mathrm{P}\left\{{ }^{1} \mathrm{H}\right\} \mathrm{NMR}\left(\mathrm{CDCl}_{3}\right): \delta 20.9(1 \mathrm{j}(\mathrm{Pt}-\mathrm{P})=4235 \mathrm{~Hz})$. MALDI-TOF (9-NA): m/z 972.82 [M] ${ }^{+}, 938.07\left[\mathrm{M}-\mathrm{Cl}^{+}\right.$. Anal. Calcd for $\mathrm{C}_{49} \mathrm{H}_{45} \mathrm{CINO}_{4}$ PPt: $\mathrm{C} 60.46, \mathrm{H}$ 4.66, N 1.44. Found: C 60.34, H 4.58, N 1.43.

1- $\left\{4^{\prime}\right.$-[(Dimethylamino)methyl]benzyl $\}$-3-ethylmaIonate (8). To a solution of $\mathbf{1}(1.50 \mathrm{~g}, 9.07 \mathrm{mmol})$ in $\mathrm{CH}_{2} \mathrm{Cl}_{2}$ $(40 \mathrm{~mL})$ at $-60^{\circ} \mathrm{C}$ was added pyridine $(0.77 \mathrm{~mL}, 9.5 \mathrm{mmol})$. A solution of ethyl mal onyl chloride $(1.2 \mathrm{~mL}, 9.5 \mathrm{mmol})$ in $\mathrm{CH}_{2-}$ $\mathrm{Cl}_{2}(25 \mathrm{~mL})$ was added dropwise, and the solution was allowed to warm overnight. The sol ution was washed with a saturated solution of $\mathrm{NaHCO}_{3}(3 \times 60 \mathrm{~mL})$, dried on $\mathrm{MgSO}_{4}$, filtered, and evaporated in vacuo, yielding 8 as a yellow oil $(2.03 \mathrm{~g}$, 80\%). ${ }^{1} \mathrm{H} N M R\left(\mathrm{CDCl}_{3}\right): \delta 7.26(\mathrm{~s}, 4 \mathrm{H}, \mathrm{Ar}-\mathrm{H}), 5.12(\mathrm{~s}, 2 \mathrm{H}$, $\left.\mathrm{OCH}_{2}\right), 4.13(\mathrm{q}, 3 \mathrm{~J}=7.5 \mathrm{~Hz}, 2 \mathrm{H}, \mathrm{OEt}), 3.37\left(\mathrm{~s}, 2 \mathrm{H}, \mathrm{CH}_{2} \mathrm{~N}\right)$, $3.36\left(\mathrm{~s}, 2 \mathrm{H}, \mathrm{C}(\mathrm{O}) \mathrm{CH}_{2} \mathrm{C}(\mathrm{O})\right), 2.18\left(\mathrm{~s}, 6 \mathrm{H}, \mathrm{NCH}_{3}\right), 1.19\left(\mathrm{t},{ }^{3} \mathrm{~J}=\right.$ $6.9 \mathrm{~Hz}, 3 \mathrm{H}, \mathrm{OEt}) .{ }^{13} \mathrm{C}\left\{{ }^{1} \mathrm{H}\right\} \mathrm{NMR}\left(\mathrm{CDCl}_{3}\right): \delta 166.2,166.1(\mathrm{CO})$, 139.0, 133.9, 129.0, $128.0(\mathrm{Ar}-\mathrm{C}), 66.7\left(\mathrm{OCH}_{2}\right), 63.7\left(\mathrm{CH}_{2} \mathrm{~N}\right)$, 61.2 (OEt), $45.1\left(\mathrm{NCH}_{3}\right), 41.3\left(\mathrm{C}(\mathrm{O}) \mathrm{CH}_{2} \mathrm{C}(\mathrm{O})\right), 13.8$ (OEt). Anal. Calcd for $\mathrm{C}_{15} \mathrm{H}_{21} \mathrm{NO}_{4}$ : C 64.50, H 7.58, N 5.01. Found: C 64.43, H 7.51, N 5.05.

1-[Bis(4-[(dimethylamino)methyl]phenyl)methyl]-3ethylmalonate (9). To a solution of ethyl malonyl chloride $(0.80 \mathrm{~mL}, 6.6 \mathrm{mmol})$ in $\mathrm{CH}_{2} \mathrm{Cl}_{2}(40 \mathrm{~mL})$ was added a solution of $4(0.96 \mathrm{~g}, 3.2 \mathrm{mmol})$ in $\mathrm{CH}_{2} \mathrm{Cl}_{2}(30 \mathrm{~mL})$. The reaction mixture was stirred for $3 \mathrm{~h}$ and evaporated in vacuo. The remaining white solid was washed with $\mathrm{Et}_{2} \mathrm{O}(4 \times 50 \mathrm{~mL})$ and suspended in $\mathrm{CH}_{2} \mathrm{Cl}_{2}(50 \mathrm{~mL})$. Then $\mathrm{NEt}_{3}(3 \mathrm{~mL})$ was added to the mixture, upon which the mixture clarified. This was washed with a saturated $\mathrm{NaHCO}_{3}$ solution $(2 \times 50 \mathrm{~mL})$ and an aqeous saturated $\mathrm{NaCl}$ solution $(50 \mathrm{~mL})$. The organic solution was dried on $\mathrm{MgSO}_{4}$, filtered, and evaporated in vacuo, yielding 9 as a yellow oil $(0.82 \mathrm{~g}, 62 \%)$. ${ }^{1} \mathrm{H} N M R\left(\mathrm{CDCl}_{3}\right): \delta 7.23(\mathrm{~s}, 8 \mathrm{H}$, $\mathrm{Ar}-\mathrm{H}), 6.87(\mathrm{~s}, \mathrm{CH}), 4.11(\mathrm{q}, 3 \mathrm{~J}=6.9 \mathrm{~Hz}, 2 \mathrm{H}, \mathrm{OEt}), 3.39$ (s, $\left.2 \mathrm{H}, \mathrm{C}(\mathrm{O}) \mathrm{CH}_{2} \mathrm{C}(\mathrm{O})\right), 3.29\left(\mathrm{~s}, 4 \mathrm{H}, \mathrm{CH}_{2} \mathrm{~N}\right), 2.15\left(\mathrm{~s}, 12 \mathrm{H}, \mathrm{NCH}_{3}\right)$, $1.15\left(\mathrm{t},{ }^{3} \mathrm{~J}=7.2 \mathrm{~Hz}, 3 \mathrm{H}, \mathrm{OEt}\right) .{ }^{13} \mathrm{C}\left\{{ }^{1} \mathrm{H}\right\} \mathrm{NMR}\left(\mathrm{CDCl}_{3}\right): \delta 165.9$, 165.2 (CO), 138.5, 138.1, 128.7, 126.7 (Ar-C), $77.3(\mathrm{OCH}), 63.6$ $\left(\mathrm{CH}_{2} \mathrm{~N}\right), 61.1$ (OEt), $45.0\left(\mathrm{NCH}_{3}\right), 41.5\left(\mathrm{C}(\mathrm{O}) \mathrm{CH}_{2} \mathrm{C}(\mathrm{O})\right), 13.7$ (OEt). Anal. Calcd for $\mathrm{C}_{24} \mathrm{H}_{32} \mathrm{~N}_{2} \mathrm{O}_{4}$ : C 69.88, H 7.82, N 6.79. Found: C 70.05, H 7.94, N 6.82.

1-\{3'-[Chloro(triphenylposphine)platino]-4'-[(dimethylamino)methyl]benzyl\}-3-ethylmalonate (10). To a solution of $3(1.26 \mathrm{~g}, 1.92 \mathrm{mmol})$ in $\mathrm{CH}_{2} \mathrm{Cl}_{2}(40 \mathrm{~mL})$ at $0{ }^{\circ} \mathrm{C}$ was added $\mathrm{NEt}_{3}(0.40 \mathrm{~mL}, 2.9 \mathrm{mmol})$ and ethyl malonyl chloride $(0.37$ $\mathrm{mL}, 2.9 \mathrm{mmol}$ ). The reaction mixture was stirred for $5 \mathrm{~h}$ and evaporated in vacuo. The remaining white solid was washed with $\mathrm{Et}_{2} \mathrm{O}(3 \times 40 \mathrm{~mL})$ and dissolved in $\mathrm{CH}_{2} \mathrm{Cl}_{2}$. The solution was washed with a saturated solution of $\mathrm{NaHCO}_{3}(2 \times 40 \mathrm{~mL})$ and a saturated solution of $\mathrm{NaCl}(2 \times 40 \mathrm{~mL})$, dried on $\mathrm{MgSO}_{4}$, and evaporated in vacuo, yielding 10 as a white solid $(0.80 \mathrm{~g}$, 54\%). ${ }^{1} \mathrm{H}$ NMR $\left(\mathrm{CDCl}_{3}\right): \delta$ 7.77-7.67 (m, 6H, PAr-H), 7.43$7.31(\mathrm{~m}, 9 \mathrm{H}, \mathrm{PAr}-\mathrm{H}), 7.01$ (d, $3 \mathrm{~J}=7.5 \mathrm{~Hz}, 1 \mathrm{H}, \mathrm{Ar}-\mathrm{H}), 6.82$ (dd, 3) $=7.5 \mathrm{~Hz}, 4 \mathrm{~J}=1.5 \mathrm{~Hz}, 1 \mathrm{H}, \mathrm{Ar}-\mathrm{H}), 6.35(\mathrm{dd}, 3 \mathrm{~J}(\mathrm{Pt}-\mathrm{H})=$

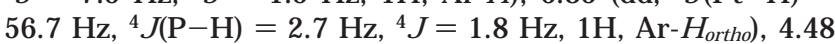
$\left(\mathrm{s}, 2 \mathrm{H}, \mathrm{OCH}_{2}\right), 4.17(\mathrm{q}, 2 \mathrm{H}, 3 \mathrm{~J}=7.3 \mathrm{~Hz}), 4.05(\mathrm{~d}, 3 \mathrm{~J}(\mathrm{Pt}-\mathrm{H})=$ $28.4 \mathrm{~Hz}$, 3) $\left.(\mathrm{P}-\mathrm{H})=2.8 \mathrm{~Hz}, 2 \mathrm{H}, \mathrm{CH}_{2} \mathrm{~N}\right), 3.12\left(\mathrm{~s}, 2 \mathrm{H}, \mathrm{C}(\mathrm{O}) \mathrm{CH}_{2} \mathrm{C}-\right.$ $(\mathrm{O})), 2.97(\mathrm{~d}, 3 \mathrm{~J}(\mathrm{Pt}-\mathrm{H})=23.8 \mathrm{~Hz}, 3 \mathrm{~J}(\mathrm{P}-\mathrm{H})=2.7 \mathrm{~Hz}, 6 \mathrm{H}$, $\left.\mathrm{NCH}_{3}\right), 1.22\left(\mathrm{t},{ }^{3} \mathrm{~J}=6.8 \mathrm{~Hz}\right) \cdot{ }^{13} \mathrm{C}\left\{{ }^{1} \mathrm{H}\right\} \mathrm{NMR}\left(\mathrm{CDCl}_{3}\right): \delta 147.66$ 
(Ar-C), $137.38\left(\mathrm{~d}, \mathrm{~J}(\mathrm{P}-\mathrm{C})=6.1 \mathrm{~Hz}, \mathrm{PAr}-\mathrm{C}_{\mathrm{ipso}}\right), 135.18(\mathrm{~d}$, 2] $\left.(\mathrm{P}-\mathrm{C})=11.0 \mathrm{~Hz}, \mathrm{Ar}-\mathrm{C}_{\text {ortho }}\right), 131.61\left(\mathrm{~d},{ }^{2} \mathrm{~J}(\mathrm{P}-\mathrm{C})=2.5 \mathrm{~Hz}\right.$, Ar-C), 130.64 (Ar-C), 130.53 (d, 4J $\left.(P-C)=2.4 \mathrm{~Hz}, \mathrm{PAr}-\mathrm{C}_{\text {para }}\right)$, $129.82,127.81\left(\mathrm{~d}, 3 \mathrm{~J}(\mathrm{P}-\mathrm{C})=11.0 \mathrm{~Hz}, \mathrm{PAr}-\mathrm{C}_{\text {meta }}\right), 122.91$ (ArC), $121.56(\mathrm{Ar}-\mathrm{C}), 74.0\left({ }^{2} \mathrm{~J}(\mathrm{Pt}-\mathrm{C})=56.3 \mathrm{~Hz}, \mathrm{CH}_{2} \mathrm{~N}\right), 67.2$ $\left(\mathrm{OCH}_{2}\right), 61.4(\mathrm{OEt}), 50.8\left(\mathrm{NCH}_{3}\right), 41.3\left(\mathrm{C}(\mathrm{O}) \mathrm{CH}_{2} \mathrm{C}(\mathrm{O})\right), 14.0$ (OEt). ${ }^{11} \mathrm{P}\left\{{ }^{1} \mathrm{H}\right\} \mathrm{NMR}\left(\mathrm{CDCl}_{3}\right): \delta 21.2\left({ }^{1} \mathrm{~J}(\mathrm{Pt}-\mathrm{P})=4236 \mathrm{~Hz}\right)$. Anal. Calcd for $\mathrm{C}_{33} \mathrm{H}_{35} \mathrm{ClNO}_{4} \mathrm{PPt}$ : C 51.40, H 4.57, N 1.82 . Found: C 51.24, H 4.65, N 1.74.

1-\{ Bis(3'-[chloro(triphenylposphine)platino]-4'-[(dimethylamino)methyl]phenyl)methyl\}-3-ethylmalonate (11). Compound $\mathbf{1 1}$ was prepared using the same method as for 10 starting from $6(2.28 \mathrm{~g}, 1.78 \mathrm{mmol})$, ethyl malonyl chloride $(0.34 \mathrm{~mL}, 2.7 \mathrm{mmol})$, and $\mathrm{NEt}_{3}(0.37 \mathrm{~mL}, 2.7 \mathrm{mmol})$, yielding 11 as a white solid (1.90 g, 76\%). ${ }^{1} \mathrm{H} \mathrm{NMR}\left(\mathrm{CDCl}_{3}\right)$ : o 7.71-7.65 (m, 12H, PAr-H ), 7.43-7.26 (m, 18H, PAr-H ), 6.67 $(\mathrm{d}, 3 \mathrm{~J}=7.5 \mathrm{~Hz}, 2 \mathrm{H}, \mathrm{Ar}-\mathrm{H}), 6.39(\mathrm{~J})(\mathrm{Pt}-\mathrm{H})=55.2 \mathrm{~Hz}, 2 \mathrm{H}, \mathrm{Ar}-$ $\left.\mathrm{H}_{\text {ortho }}\right), 5.79$ (dd, $\left.{ }^{3} \mathrm{~J}=7.8 \mathrm{~Hz},{ }^{4} \mathrm{~J}=0.9 \mathrm{~Hz}, 2 \mathrm{H}, \mathrm{Ar}-\mathrm{H}\right), 5.47$ (s, $1 \mathrm{H}, \mathrm{OCH}), 4.08(\mathrm{q}, 2 \mathrm{H}, 3 \mathrm{~J}=6.9 \mathrm{~Hz}, \mathrm{OEt}), 4.01 / 3.95(\mathrm{dd}, \mathrm{AB}$, 3) $=13.5 \mathrm{~Hz}$, 3 $\left.(\mathrm{P}-\mathrm{H})=2.7 \mathrm{~Hz}, 4 \mathrm{H}, \mathrm{CH}_{2} \mathrm{~N}\right), 2.96,2.93(2 \times \mathrm{d}$, 3) $\left.(\mathrm{P}-\mathrm{H})=2.7 \mathrm{~Hz}, 12 \mathrm{H}, \mathrm{NCH}_{3}\right), 2.46\left(\mathrm{~s}, 2 \mathrm{H}, \mathrm{C}(\mathrm{O}) \mathrm{CH}_{2} \mathrm{C}(\mathrm{O})\right)$, 1.15 (t, 3j $=6.9 \mathrm{~Hz}, \mathrm{OEt}) .{ }^{13} \mathrm{C}\left\{{ }^{1} \mathrm{H}\right\} \mathrm{NMR}\left(\mathrm{CDCl}_{3}\right): \delta 146.47$ $\left.(\operatorname{Ar}-C), 136.64(d, 1](P-C)=6.7 \mathrm{~Hz}, P A r-C_{i p s o}\right), 136.47(d$, 2 $\mathrm{f}(\mathrm{P}-\mathrm{C})=2.4 \mathrm{~Hz}, \mathrm{Ar}-\mathrm{C}), 135.12(\mathrm{~d}$, 光 $(\mathrm{P}-\mathrm{C})=10.9 \mathrm{~Hz}, \mathrm{PAr}-$ $\mathrm{C}_{\text {ortho }}$ ), 130.8 (Ar-C), 130.46 (d, 4 $\left.(\mathrm{P}-\mathrm{C})=1.8 \mathrm{~Hz}, \mathrm{Ar}-\mathrm{C}_{\mathrm{para}}\right)$, $\left.130.03(\mathrm{Ar}-\mathrm{C}), 127.80(\mathrm{~d}, 3)(\mathrm{P}-\mathrm{C})=11.6 \mathrm{~Hz}, \mathrm{PAr}-\mathrm{C}_{\text {meta }}\right), 121.63$ (Ar-C), $121.29(\mathrm{Ar}-\mathrm{C}), 77.8(\mathrm{OCH}), 74.1\left({ }^{2} \mathrm{~J}(\mathrm{Pt}-\mathrm{C})=49.7 \mathrm{~Hz}\right.$, $\left.\mathrm{CH}_{2} \mathrm{~N}\right), 61.1(\mathrm{OEt}), 50.9,50.8\left(\mathrm{NCH}_{3}\right), 40.8\left(\mathrm{C}(\mathrm{O}) \mathrm{CH}_{2} \mathrm{C}(\mathrm{O})\right), 14.0$ (OEt). ${ }^{31} \mathrm{P}\left\{{ }^{1} \mathrm{H}\right\} N M R\left(\mathrm{CDCl}_{3}\right): \delta 21.6\left({ }^{1} \mathrm{~J}(\mathrm{Pt}-\mathrm{P})=4262 \mathrm{~Hz}\right)$. Anal. Calcd for $\mathrm{C}_{60} \mathrm{H}_{60} \mathrm{Cl}_{2} \mathrm{~N}_{2} \mathrm{O}_{4} \mathrm{P}_{2} \mathrm{Pt}_{2}$ : C 51.62, $\mathrm{H}$ 4.33, N 2.01 . Found: C 51.69, H 4.28, N 1.97.

1-\{4'-[(Dimethylamino)methyl]-3'-[iodo(triphenylposphine)platino]benzyl\}-3-ethylmalonate (12). To a sol ution of $10(0.44 \mathrm{~g}, 0.57 \mathrm{mmol})$ in acetone $(25 \mathrm{~mL})$ was added $\mathrm{Nal}$ $(0.85 \mathrm{~g}, 4.4 \mathrm{mmol})$. The reaction mixture was stirred for $2 \mathrm{~h}$ and evaporated in vacuo. The resultant white solid was extracted with $\mathrm{CH}_{2} \mathrm{Cl}_{2}(20 \mathrm{~mL})$. Hexanes $(60 \mathrm{~mL})$ were added to the solution, whereupon a precipitate was formed. This was isolated and dried in vacuo, giving $\mathbf{1 2}$ as a tan solid $(0.40 \mathrm{~g}$, 81\%). ${ }^{1} \mathrm{H}$ NMR $\left(\mathrm{CDCl}_{3}\right): \delta$ 7.77-7.69 (m, 6H, PAr-H), 7.44$7.31(\mathrm{~m}, 9 \mathrm{H}, \mathrm{PAr}-\mathrm{H}), 7.02(\mathrm{~d}, 3 \mathrm{~J}=7.5 \mathrm{~Hz}, 1 \mathrm{H}, \mathrm{Ar}-\mathrm{H}), 6.86(\mathrm{dd}$, 3) $=7.8 \mathrm{~Hz}, 4 \mathrm{~J}=1.8 \mathrm{~Hz}, 1 \mathrm{H}, \mathrm{Ar}-\mathrm{H}), 6.35(\mathrm{dd}, 3 \mathrm{~J}(\mathrm{Pt}-\mathrm{H})=$ $58.2 \mathrm{~Hz}$, 少 $\left.(\mathrm{P}-\mathrm{H})=3.6 \mathrm{~Hz},{ }^{4} \mathrm{~J}=1.5 \mathrm{~Hz}, 1 \mathrm{H}, \mathrm{Ar}-\mathrm{H}_{\text {ortho }}\right), 4.48$ $\left(\mathrm{s}, 2 \mathrm{H}, \mathrm{OCH}_{2}\right), 4.17(\mathrm{q}, 2 \mathrm{H}, 3 \mathrm{j}=6.9 \mathrm{~Hz}), 4.09(\mathrm{~d}, 3 \mathrm{j}(\mathrm{Pt}-\mathrm{H})=$ $24.0 \mathrm{~Hz}$, 3 $\left.(\mathrm{P}-\mathrm{H})=2.4 \mathrm{~Hz}, 2 \mathrm{H}, \mathrm{CH}_{2} \mathrm{~N}\right), 3.15(\mathrm{~d}$, 3 $(\mathrm{Pt}-\mathrm{H})=$ $\left.24.6 \mathrm{~Hz},{ }^{3} \mathrm{~J}(\mathrm{P}-\mathrm{H})=3.0 \mathrm{~Hz}, 6 \mathrm{H}, \mathrm{NCH}_{3}\right), 3.12\left(\mathrm{~s}, 2 \mathrm{H}, \mathrm{C}(\mathrm{O}) \mathrm{CH}_{2} \mathrm{C}-\right.$ $(\mathrm{O})), 1.23\left(\mathrm{t},{ }^{3} \mathrm{~J}=6.9 \mathrm{~Hz}, \mathrm{OEt}\right) \cdot{ }^{13} \mathrm{C}\left\{{ }^{1} \mathrm{H}\right\} \mathrm{NMR}\left(\mathrm{CDCl}_{3}\right): \delta 147.64$ $(\operatorname{Ar}-\mathrm{C}), 136.35\left(\mathrm{~d},{ }^{1} \mathrm{~J}(\mathrm{P}-\mathrm{C})=6.7 \mathrm{~Hz}, \operatorname{PAr}-\mathrm{C}_{\mathrm{ipso}}\right), 135.31(\mathrm{~d}$, 2) $\left.(P-C)=10.4 \mathrm{~Hz}, A r-C_{\text {ortho }}\right), 132.28(\mathrm{Ar}-\mathrm{C}), 131.62(\mathrm{~d}$, $4 \mathrm{~J}(\mathrm{P}-$ C) $=2.5 \mathrm{~Hz}, \mathrm{Ar}-\mathrm{C}), 130.46(\mathrm{Ar}-\mathrm{C}), 130.60(\mathrm{~d}, 4 \mathrm{~J}(\mathrm{P}-\mathrm{C})=3.3$ $\left.\mathrm{Hz}, \mathrm{Ar}-\mathrm{C}_{\text {para }}\right), 127.76$ (d, 3) $\left.(\mathrm{P}-\mathrm{C})=11.5 \mathrm{~Hz}, \mathrm{PAr}-\mathrm{C}_{\text {meta }}\right), 123.14$ $(\mathrm{Ar}-\mathrm{C}), 121.80$ ( $\mathrm{3} \mathrm{J}(\mathrm{Pt}-\mathrm{C})=32.8 \mathrm{~Hz}, \mathrm{Ar}-\mathrm{C}), 74.2\left({ }^{2} \mathrm{~J}(\mathrm{Pt}-\mathrm{C})=\right.$ $\left.53.4 \mathrm{~Hz}, \mathrm{CH}_{2} \mathrm{~N}\right), 67.2\left(\mathrm{OCH}_{2}\right), 61.5(\mathrm{OEt}), 53.2\left(\mathrm{NCH}_{3}\right), 41.4$ $\left(\mathrm{C}(\mathrm{O}) \mathrm{CH}_{2} \mathrm{C}(\mathrm{O})\right), 14.1$ (OEt). ${ }^{31} \mathrm{P}\left\{{ }^{1} \mathrm{H}\right\} \mathrm{NMR}\left(\mathrm{CDCl}_{3}\right): \delta 19.7$ (1) $(\mathrm{Pt}-\mathrm{P})=4189 \mathrm{~Hz}$ ). Anal. Calcd for $\mathrm{C}_{33} \mathrm{H}_{35} \mathrm{INO}_{4} \mathrm{PPt}$ : C 45.95, H 4.09, N 1.62. Found: C 45.80, H 3.98, N 1.58.

1- $\{$ Bis(4'-[(dimethylamino)methyl]-3'-[iodo(triphenylposphine)platino]phenyl)methyl\}-3-ethylmalonate (13). To a solution of 11 (1.43 g, $1.02 \mathrm{mmol})$ in acetone $(50 \mathrm{~mL})$ was added $\mathrm{Nal}(1.5 \mathrm{~g}, 10 \mathrm{mmol})$. The reaction mixture was stirred for $2 \mathrm{~h}$ and evaporated in vacuo, and the remaining solid was extracted with $\mathrm{CH}_{2} \mathrm{Cl}_{2}(50 \mathrm{~mL})$. This was evaporated in vacuo, yielding 13 as a tan solid (1.38 g, 86\%). ${ }^{1} \mathrm{H} \mathrm{NMR}\left(\mathrm{CDCl}_{3}\right): \delta$ 7.72-7.63 (m, 12H, PAr-H), 7.38-7.27 (m, 18H, PAr-H), 6.68 $(\mathrm{d}, 3 \mathrm{~J}=7.8 \mathrm{~Hz}, 2 \mathrm{H}, \mathrm{Ar}-\mathrm{H}), 6.39\left(\mathrm{dd},{ }^{3} \mathrm{~J}(\mathrm{Pt}-\mathrm{H})=59.8 \mathrm{~Hz},{ }^{4}\right)(\mathrm{P}-$ $\left.\mathrm{H})=3.5 \mathrm{~Hz},{ }^{4} \mathrm{~J}=1.2 \mathrm{~Hz}, 2 \mathrm{H}, \mathrm{Ar}-\mathrm{H}_{\text {ortho }}\right), 5.70$ (dd, 3J $=7.6 \mathrm{~Hz}$, 4) $=1.2 \mathrm{~Hz}, 2 \mathrm{H}, \mathrm{Ar}-\mathrm{H}), 5.52(\mathrm{~s}, 1 \mathrm{H}, \mathrm{OCH}), 4.08(\mathrm{q}, 2 \mathrm{H}, 3 \mathrm{~J}=$ $7.5 \mathrm{~Hz}, \mathrm{OEt}$ ), 4.05/3.99 (dd, $A B,{ }^{3} \mathrm{~J}=13.4 \mathrm{~Hz}, 3 \mathrm{~J}(\mathrm{P}-\mathrm{H})=3.0$ $\left.\mathrm{Hz}, 4 \mathrm{H}, \mathrm{CH}_{2} \mathrm{~N}\right), 3.15,3.09(2 \times \mathrm{d}$, 3 $(\mathrm{P}-\mathrm{H})=2.7 \mathrm{~Hz}, 12 \mathrm{H}$, $\left.\mathrm{NCH}_{3}\right), 2.52\left(\mathrm{~s}, 2 \mathrm{H}, \mathrm{C}(\mathrm{O}) \mathrm{CH}_{2} \mathrm{C}(\mathrm{O})\right), 1.15$ (t, 3 $\left.3=6.9 \mathrm{~Hz}, \mathrm{OEt}\right)$. ${ }^{13} \mathrm{C}\left\{{ }^{1} \mathrm{H}\right\} \mathrm{NMR}\left(\mathrm{CDCl}_{3}\right): \delta 166.04,164.43(\mathrm{CO}), 146.45$ (Ar-C), $136.22(\mathrm{~d}, 2 \mathrm{~J}(\mathrm{P}-\mathrm{C})=2.4 \mathrm{~Hz}, \mathrm{Ar}-\mathrm{C}), 135.13(\mathrm{~d}$, 4 $\mathrm{J}(\mathrm{P}-\mathrm{C})=11.0$ $\left.\mathrm{Hz}, \mathrm{PAr}-\mathrm{C}_{\text {ortho }}\right), 136.64$ (d, IJ $\left.(\mathrm{P}-\mathrm{C})=6.7 \mathrm{~Hz}, \mathrm{PAr}-\mathrm{C}_{\mathrm{ipso}}\right), 132.4$ (3) $(\mathrm{Pt}-\mathrm{C})=29.0 \mathrm{~Hz}, \mathrm{Ar}-\mathrm{C}), 131.67$ (3) $(\mathrm{Pt}-\mathrm{C})=30.4 \mathrm{~Hz}, \mathrm{Ar}-$ C), 130.34 (PAr-C para $), 127.69\left(\mathrm{~d},{ }^{3}\right)(\mathrm{P}-\mathrm{C})=10.9 \mathrm{~Hz}, \mathrm{PAr}-$ $\left.\mathrm{C}_{\text {meta }}\right), 121.85$ (Ar-C), 121.14 (Ar-C), 74.1 ( ${ }^{2} \mathrm{~J}(\mathrm{Pt}-\mathrm{C})=46.7 \mathrm{~Hz}$, $\left.\mathrm{CH}_{2} \mathrm{~N}\right), 61.1$ (OEt), 53.3, 52.9 $\left(\mathrm{NCH}_{3}\right), 40.9\left(\mathrm{C}(\mathrm{O}) \mathrm{CH}_{2} \mathrm{C}(\mathrm{O})\right), 14.0$ (OEt). ${ }^{31} \mathrm{P}\left\{{ }^{1} \mathrm{H}\right\} N M R\left(\mathrm{CDCl}_{3}\right): \delta 20.0\left({ }^{1} \mathrm{~J}(\mathrm{Pt}-\mathrm{P})=4202 \mathrm{~Hz}\right)$. Anal. Calcd for $\mathrm{C}_{60} \mathrm{H}_{60} \mathrm{O}_{2} \mathrm{~N}_{2} \mathrm{O}_{4} \mathrm{P}_{2} \mathrm{Pt}_{2}$ : C 45.64, H 3.83, N 1.77 . Found: C 45.88, H 3.76, N 1.85.

1,2-Dihydro-61-ethoxycarbonyl-61- $\{$ 4-[(dimethylamino)methyl]benzyloxycarbonyl\}-1,2-methano[60]fullerene

(14). To a solution of $8(0.38 \mathrm{~g}, 1.3 \mathrm{mmol}), \mathrm{C}_{60}(0.91 \mathrm{~g}, 1.3$ $\mathrm{mmol})$, and $\mathrm{I}_{2}(0.65 \mathrm{~g}, 2.5 \mathrm{mmol})$ in toluene $(900 \mathrm{~mL})$ was added dropwise DBU (1.1 mL, $7.1 \mathrm{mmol})$. The reaction mixture was stirred for $17 \mathrm{~h}$ and poured on top of a silica gel column. Elution with toluene afforded $0.20 \mathrm{~g}$ of unreacted $\mathrm{C}_{60}$. E lution with toluene/MeOH (95:5 v/v \%) gave the crude product after evaporation of the solvent. This was dissolved in $\mathrm{CS}_{2}(10 \mathrm{~mL})$ and pentane was added $(75 \mathrm{~mL})$, whereupon the product preci pitated. This procedure was repeated and the precipitate dried in vacuo, yielding 14 as a light brown solid $(0.44 \mathrm{~g}, 35 \%$, $45 \%$ based on reacted $\left.\mathrm{C}_{60}\right)$. ${ }^{1} \mathrm{H}$ NMR $\left(\mathrm{CS}_{2} / \mathrm{C}_{6} \mathrm{D}_{6}, 3: 1 \mathrm{v} / \mathrm{v} \%\right): \delta$ $\left.7.23\left(\mathrm{~d}, 3^{3}\right)=8.4 \mathrm{~Hz}, 2 \mathrm{H}, \mathrm{Ar}-\mathrm{H}\right), 7.21\left(\mathrm{~d},{ }^{3} \mathrm{~J}=8.4 \mathrm{~Hz}, 2 \mathrm{H}, \mathrm{Ar}-\right.$ $\mathrm{H}), 5.25\left(\mathrm{~s}, 2 \mathrm{H}, \mathrm{OCH}_{2}\right), 4.21$ (q, $\left.{ }^{3} \mathrm{~J}=7.2 \mathrm{~Hz}, 2 \mathrm{H}, \mathrm{OEt}\right), 3.27$ (s, $2 \mathrm{H}, \mathrm{CH}_{2} \mathrm{~N}$ ), $2.09\left(\mathrm{~s}, 6 \mathrm{H}, \mathrm{NCH}_{3}\right), 1.14$ (t, 3 $=7.2 \mathrm{~Hz}, 3 \mathrm{H}, \mathrm{OEt}$ ). ${ }^{13} \mathrm{C}\left\{{ }^{1} \mathrm{H}\right\}$ NMR $\left(\mathrm{CS}_{2} / \mathrm{C}_{6} \mathrm{D}_{6}, 3: 1 \mathrm{v} / \mathrm{v} \%\right): \delta 162.73,162.64(\mathrm{CO})$, $144.63,145.48,145.46,145.38,145.07,144.96,144.91,144.87$, $144.82,144.14,143.31,143.25,142.48,142.09,141.19,141.17$, $140.62,139.56,139.38,133.67,129.24,129.17\left(\mathrm{C}_{60}-\mathrm{C}\right.$ and $\mathrm{Ar}-$ C), $71.9\left(\mathrm{C}_{60}-\mathrm{sp}^{3}\right), 68.6,\left(\mathrm{OCH}_{2}\right), 64.2\left(\mathrm{CH}_{2} \mathrm{~N}\right), 63.2(\mathrm{OEt}), 52.2$ $\left(\mathrm{C}(\mathrm{O}) \mathrm{CH}_{2} \mathrm{C}(\mathrm{O})\right), 45.6\left(\mathrm{NCH}_{3}\right), 14.4$ (OEt). MALDI-TOF (9$\mathrm{NA}): \mathrm{m} / \mathrm{z} 997.0\left([\mathrm{M}]^{+}\right)$. Anal. Calcd for $\mathrm{C}_{75} \mathrm{H}_{19} \mathrm{NO}_{4}$ : C 90.26, H 1.92, N 1.40. Found: C 90.05, H 1.86, N 1.32.

1,2-Dihydro-61-ethoxycarbonyl-61- $\left\{\right.$ bis $\left\{4^{\prime}\right.$-[(dimethylamino)methyl]phenyl \} methyl oxycarbonyl \}-1,2methano[60]fullerene (15). To a solution of $9(0.41 \mathrm{~g}, 0.99$ $\mathrm{mmol}), \mathrm{C}_{60}(0.86 \mathrm{~g}, 1.2 \mathrm{mmol})$, and $\mathrm{I}_{2}(0.51 \mathrm{~g}, 2.0 \mathrm{mmol})$ in toluene $(900 \mathrm{~mL})$ was added DBU $(0.90 \mathrm{~mL}, 7.0 \mathrm{mmol})$ dropwise. The reaction mixture was stirred for $17 \mathrm{~h}$ and poured on top of a silica gel column. Elution with toluene afforded $0.23 \mathrm{~g}$ of unreacted $\mathrm{C}_{60}$. Elution with toluene/MeOH $(95: 5 \mathrm{v} / \mathrm{V}$ $\%)$ gave the crude product after evaporation of the solvent. This was dissolved in $\mathrm{CS}_{2}(10 \mathrm{~mL})$, and acetone was added (75 $\mathrm{mL}$ ), whereupon the product precipitated. This was isolated, washed with acetone $(40 \mathrm{~mL})$, and dried in vacuo, yielding 15 as a light brown solid $(0.53 \mathrm{~g}, 47 \%, 54 \%$ based on reacted $\left.\mathrm{C}_{60}\right)$. ${ }^{1 \mathrm{H}} \mathrm{NMR}\left(\mathrm{CDCl}_{3}\right): \delta 7.41\left(\mathrm{~d},{ }^{3 \mathrm{j}}=8.1 \mathrm{~Hz}, 4 \mathrm{H}\right.$, $\operatorname{Ar}-\mathrm{H}), 7.31(\mathrm{~d}, 3 \mathrm{~J}=8.1 \mathrm{~Hz}, 4 \mathrm{H}, \mathrm{Ar}-\mathrm{H}), 7.26(\mathrm{~s}, 1 \mathrm{H}, \mathrm{OCH}), 4.51$ $\left(\mathrm{q},{ }^{3} \mathrm{~J}=7.5 \mathrm{~Hz}, 2 \mathrm{H}, \mathrm{OEt}\right), 3.41\left(\mathrm{~s}, 4 \mathrm{H}, \mathrm{CH}_{2} \mathrm{~N}\right), 2.23(\mathrm{~s}, 12 \mathrm{H}$, $\left.\mathrm{NCH}_{3}\right), 1.14$ (t, $\left.{ }^{3} \mathrm{~J}=7.2 \mathrm{~Hz}, 3 \mathrm{H}, \mathrm{OEt}\right) .{ }^{13} \mathrm{C}\left\{{ }^{1} \mathrm{H}\right\} \mathrm{NMR}\left(\mathrm{CDCl}_{3}\right)$ : $\delta 163.51,162.72$ (CO), 145.30, 145.26, 145.20, 145.17, 145.15, $145.03,144.91,144.91,144.88,144.71,144.69,144.67,144.63$, $144.46,143.89,143.83,143.06,143.01,142.98,142.97,142.22$, $142.21,140.94,142.21,141.86,140.94,140.90,139.63,139.36$, 138.51, 137.61, 129.16, $127.62\left(\mathrm{C}_{60}-\mathrm{C}\right.$ and $\left.\mathrm{Ar}-\mathrm{C}\right), 79.7(\mathrm{OCH})$, $71.5\left(\mathrm{C}_{60}-\mathrm{Sp}^{3}\right), 64.0\left(\mathrm{CH}_{2} \mathrm{~N}\right), 63.5(\mathrm{OEt}), 52.1\left(\mathrm{C}(\mathrm{O}) \mathrm{CH}_{2} \mathrm{C}(\mathrm{O})\right)$, $45.5\left(\mathrm{NCH}_{3}\right), 14.2$ (OEt). MALDI-TOF (9-NA): m/z 1131.5 $\left([\mathrm{M}]^{+}\right)$. Anal. Calcd for $\mathrm{C}_{84} \mathrm{H}_{30} \mathrm{~N}_{2} \mathrm{O}_{4}$ : C 89.19, H 2.67, N 2.48 . Found: C 89.28, H 2.57, N 2.50.

1,2-Dihydro-61-ethoxycarbonyl-61- $\{3$ '-[chloro(DMSO)platino]-4'-[(dimethylamino)methyl]benzyloxycarbonyl \}1,2-methano[60]fullerene (16). To a sol ution of $\mathbf{1 4}$ (227 mg, $0.227 \mathrm{mmol})$ in 1,2-dichlorobenzene $(50 \mathrm{~mL})$ was added cis$\mathrm{PtCl}_{2}(\mathrm{DMSO})_{2}(101 \mathrm{mg}, 0.239 \mathrm{mmol})$ and a solution of $\mathrm{NaOAC}$ $(20 \mathrm{mg}, 0.24 \mathrm{mmol})$ in $\mathrm{MeOH}(20 \mathrm{~mL})$. The reaction mixture was heated at $65{ }^{\circ} \mathrm{C}$ for $17 \mathrm{~h}$ and evaporated in vacuo. The crude product was extracted with $\mathrm{CS}_{2}(40 \mathrm{~mL})$, filtered, and evaporated in vacuo. The product was washed with $\mathrm{MeOH}(2$ $\times 40 \mathrm{~mL})$ and $\mathrm{Et}_{2} \mathrm{O}(2 \times 40 \mathrm{~mL})$ and dried in vacuo, yielding 16 as a brown solid (168 mg, 57\%). ${ }^{1} \mathrm{H} \mathrm{NMR}\left(\mathrm{CDCl}_{3}\right): \delta 8.10$ $\left(\mathrm{d},{ }^{3} \mathrm{~J}(\mathrm{Pt}-\mathrm{H})=45.9 \mathrm{~Hz},{ }^{4} \mathrm{~J}=1.2 \mathrm{~Hz}, 1 \mathrm{H}, \mathrm{Ar}-\mathrm{H}_{\text {ortho }}\right), 7.26$ (dd, 


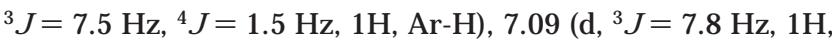
$\mathrm{Ar}-\mathrm{H}), 4.53\left(\mathrm{q},{ }^{3} \mathrm{~J}=7.2 \mathrm{~Hz}, 2 \mathrm{H}, \mathrm{OEt}\right), 4.00\left(\mathrm{~s},{ }^{3} \mathrm{~J}(\mathrm{Pt}-\mathrm{H})=38.1\right.$ $\left.\mathrm{Hz}, 2 \mathrm{H}, \mathrm{CH}_{2} \mathrm{~N}\right), 3.55\left(\mathrm{~s}\right.$, 3 $\left.(\mathrm{Pt}-\mathrm{H})=25.6 \mathrm{~Hz}, 6 \mathrm{H}, \mathrm{SCH}_{3}\right), 2.94$ $\left(\mathrm{s},{ }^{3} \mathrm{f}(\mathrm{Pt}-\mathrm{H})=32.4 \mathrm{~Hz}, 6 \mathrm{H}, \mathrm{NCH}_{3}\right), 1.43\left(\mathrm{t},{ }^{3} \mathrm{~J}=6.9 \mathrm{~Hz}, 3 \mathrm{H}\right.$, OEt). ${ }^{13} \mathrm{C}\left\{{ }^{1} \mathrm{H}\right\}$ NMR $\left(\mathrm{CDCl}_{3}\right): \delta 163.51,163.48(\mathrm{CO}), 146.89$, $145.41,145.28,145.24,145.20,145.17,145.14,144.85,144.68$, $144.65,144.57,144.43,143.86,143.84,143.05,143.01,142.96$, $142.93,142.22,142.19,141.91,141.89,140.91,140.79,139.25$, 138.91, 136.44, 135.08 ( $\mathrm{J}(\mathrm{Pt}-\mathrm{C})=52.3 \mathrm{~Hz}), 132.16,130.53$, 127.70, 125.78, 121.69 (3) (Pt-C) = $36.9 \mathrm{~Hz}$ ), 74.7 ( ${ }^{2} \mathrm{~J}(\mathrm{Pt}-\mathrm{C})$ $\left.=53.8 \mathrm{~Hz}, \mathrm{CH}_{2} \mathrm{~N}\right), 71.6\left(\mathrm{C}_{60}-\mathrm{Sp}^{3}\right), 69.7\left(\mathrm{OCH}_{2}\right), 63.4(\mathrm{OEt}), 52.3$ $\left(\mathrm{NCH}_{3}\right), 46.8$ ( $\left.{ }^{2} \mathrm{~J}(\mathrm{Pt}-\mathrm{C})=60.8 \mathrm{~Hz}, \mathrm{SCH}_{3}\right), 14.2$ (OEt). Anal. Calcd for $\mathrm{C}_{77} \mathrm{H}_{24} \mathrm{CINO}_{5} \mathrm{PtS}$ : C 70.83, $\mathrm{H}$ 1.85, N 1.07. Found: C 70.76, H 4.64, N 1.14.

1,2-Dihydro-61-ethoxycarbonyl-61- $\left\{4^{\prime}\right.$-[(dimethylamino)methyl]-3'-[iodo(triphenylposphine)platino]benzyloxycarbonyl\}-1,2-methano[60]fullerene (17). To a solution of 14 (173 mg, $0.200 \mathrm{mmol}), \mathrm{C}_{60}\left(208 \mathrm{mg}, 0.288 \mathrm{mmol}\right.$ ), and $\mathrm{I}_{2}$ (100 $\mathrm{mg}, 0.388 \mathrm{mmol})$ in toluene $(200 \mathrm{~mL})$ was added DBU $(0.12 \mathrm{~mL}, 0.80 \mathrm{mmol})$ dropwise. The reaction mixture was stirred for $17 \mathrm{~h}$ and poured on top of a silica gel column. A purple-colored fraction was eluted with toluene, affording 0.12 $\mathrm{g}$ of unreacted $\mathrm{C}_{60}$. Elution with toluene/MeOH $(95: 5 \mathrm{v} / \mathrm{v} \%$, red-col ored fraction) gave the crude product after evaporation of the solvent. This was washed with acetone $(40 \mathrm{~mL})$ and dried in vacuo, yielding 17 as a light brown solid (118 mg, 37\%, $60 \%$ based on reacted $\left.\mathrm{C}_{60}\right)$. ${ }^{1} \mathrm{H}$ NMR $\left(\mathrm{CDCl}_{3}\right): \delta 7.78-7.72(\mathrm{~m}$, $6 \mathrm{H}, \mathrm{PAr}-\mathrm{H}), 7.42-7.33(\mathrm{~m}, 9 \mathrm{H}, \mathrm{PAr}-\mathrm{H}), 7.08\left(\mathrm{~d},{ }^{3} \mathrm{~J}=7.7 \mathrm{~Hz}\right.$, $1 \mathrm{H}, \mathrm{Ar}-\mathrm{H}), 7.04\left(\mathrm{~d},{ }^{3} \mathrm{~J}=8.2 \mathrm{~Hz}, 1 \mathrm{H}, \mathrm{Ar}-\mathrm{H}\right), 6.40(\mathrm{~d}, 3 \mathrm{~J}(\mathrm{Pt}-\mathrm{H})$

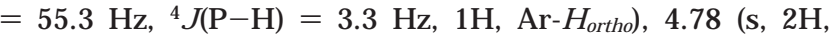
$\left.\mathrm{OCH}_{2}\right), 4.44(\mathrm{q}, 2 \mathrm{H}, 3 \mathrm{~J}=7.1 \mathrm{~Hz}), 4.12(\mathrm{~d}, 3 \mathrm{~J}(\mathrm{Pt}-\mathrm{H})=26.1 \mathrm{~Hz}$, 3 $\left.(\mathrm{P}-\mathrm{H})=2.7 \mathrm{~Hz}, 2 \mathrm{H}, \mathrm{CH}_{2} \mathrm{~N}\right), 3.17\left(\mathrm{~d},{ }^{3} \mathrm{f}(\mathrm{Pt}-\mathrm{H})=24.6 \mathrm{~Hz}\right.$, 3) $\left.(\mathrm{P}-\mathrm{H})=2.7 \mathrm{~Hz}, 6 \mathrm{H}, \mathrm{NCH}_{3}\right), 1.31$ (t, 3) $\left.=7.6 \mathrm{~Hz}, \mathrm{OEt}\right) .{ }^{13} \mathrm{C}-$ $\left\{{ }^{1} \mathrm{H}\right\} \mathrm{NMR}\left(\mathrm{CDCl}_{3}\right): \delta 163.36,163.26(\mathrm{CO}), 148.08,145.28$, $145.26,145.23,145.20,145.14,145.13,144.90,144.71,144.69$, $144.62,144.58,144.55,143.89,143.10,143.06,143.04,143.00$, $142.24,20,141.93,141.81,140.98,140.89,139.06,135.32$ (d, 4f $\left.(\mathrm{P}-\mathrm{C})=10.4 \mathrm{~Hz}, \mathrm{PAr}-\mathrm{C}_{\text {ortho }}\right), 132.18,130.62\left(\mathrm{PAr}-\mathrm{C}_{\text {para }}\right)$, $128.02,127.84\left(\mathrm{~d}, 3 \mathrm{~J}(\mathrm{P}-\mathrm{C})=11.0 \mathrm{~Hz}, \mathrm{PAr}-\mathrm{C}_{\text {meta }}\right), 123.71$, 121.97, $74.2\left(\mathrm{CH}_{2} \mathrm{~N}\right), 71.6\left(\mathrm{C}_{60}-\mathrm{Sp}^{3}\right), 69.0\left(\mathrm{OCH}_{2}\right), 63.3(\mathrm{OEt})$, $53.2\left(\mathrm{NCH}_{3}\right), 14.1$ (OEt). ${ }^{31} \mathrm{P}\left\{{ }^{1} \mathrm{H}\right\} \mathrm{NMR}\left(\mathrm{CDCl}_{3}\right): \delta 20.1$ (1) (Pt$\mathrm{P})=4167 \mathrm{~Hz})$. MALDI-TOF (9-NA): m/z $1454.0\left([\mathrm{M}-\mathrm{I}]^{+}\right)$. Anal. Calcd for $\mathrm{C}_{93} \mathrm{H}_{33} \mathrm{NNO}_{4} \mathrm{PPt}$ : C 70.64, H 2.10, N 0.89 . Found: C 70.46, H 2.11, N 0.86.

1,2-Dihydro-61-ethoxycarbonyl-61- $\left\{\right.$ bis $\left\{4^{\prime}\right.$-[(dimethylamino)methyl]-3'-[iodo(triphenylposphine)platino]\}methyloxycarbonyl\}-1,2-methano[60]fullerene (18). To a solution of 15 (346 mg, $0.219 \mathrm{mmol}$ ), $\mathrm{C}_{60}$ (205 mg, $0.285 \mathrm{mmol}$ ), and $\mathrm{I}_{2}(100 \mathrm{mg}, 0.388 \mathrm{mmol})$ in toluene $(200 \mathrm{~mL})$ was added DBU $(0.12 \mathrm{~mL}, 0.80 \mathrm{mmol})$ dropwise. The reaction mixture was stirred for $17 \mathrm{~h}$ and poured on top of a silica gel column. A purple-colored fraction was eluted with toluene, affording $116 \mathrm{mg}$ of unreacted $\mathrm{C}_{60}$. Elution with toluene/MeOH $(95: 5 \mathrm{v} / \mathrm{V}$ $\%$, red-colored fraction) gave the crude product after evaporation of the solvent. This was washed with hexanes $(40 \mathrm{~mL})$ and dried in vacuo, yiel ding $\mathbf{1 8}$ as a light brown solid (168 mg, $33 \%, 59 \%$ based on reacted $\left.\mathrm{C}_{60}\right)$. ${ }^{1} \mathrm{H}$ NMR $\left(\mathrm{CDCl}_{3}\right): \delta 7.64-$ $7.57(\mathrm{~m}, 12 \mathrm{H}, \mathrm{PAr}-\mathrm{H}), 7.26-7.23(\mathrm{~m}, 18 \mathrm{H}, \mathrm{PAr}-\mathrm{H}), 6.77\left(\mathrm{~d},{ }^{3} \mathrm{~J}\right.$ $=7.2 \mathrm{~Hz}, 2 \mathrm{H}, \mathrm{Ar}-\mathrm{H}), 6.34(\mathrm{~d}, 3 \mathrm{~J}=7.2 \mathrm{~Hz}, 2 \mathrm{H}, \mathrm{Ar}-\mathrm{H}), 6.39(\mathrm{~s}$, $\left.2 \mathrm{H}, \mathrm{Ar}-\mathrm{H}_{\text {ortho }}\right), 5.97(\mathrm{~s}, 1 \mathrm{H}, \mathrm{OCH}), 4.36(\mathrm{q}, 2 \mathrm{H}, 3 \mathrm{~J}=7.2 \mathrm{~Hz}$, OEt), 4.25/3.90 (AB, J (AB) $\left.=13.8 \mathrm{~Hz}, 4 \mathrm{H}, \mathrm{CH}_{2} \mathrm{~N}\right), 3.27,3.97$ $\left(2 \times \mathrm{d}, 3 \mathrm{~J}(\mathrm{P}-\mathrm{H})=2.7 \mathrm{~Hz}, 12 \mathrm{H}, \mathrm{NCH}_{3}\right), 1.23(\mathrm{t}, 3 \mathrm{j}=6.9 \mathrm{~Hz}$, OEt). ${ }^{13} \mathrm{C}\left\{{ }^{1} \mathrm{H}\right\}$ NMR $\left(\mathrm{CDCl}_{3}\right): \delta 163.27,161.88(\mathrm{CO}), 147.04$, $145.35,145.24,145.15,145.11,144.98,144.84,144.75,144.64$, $144.58,144.55,144.39,144.34,143.86,143.80,143.10,143.09$, 143.05, 142.96, 142.91, 142.21, 142.13, 141.88, 144.66, 140.92, $140.69,139.42,138.62,135.95\left(\mathrm{~d}, 1 \mathrm{~J}(\mathrm{P}-\mathrm{C})=6.2 \mathrm{~Hz}, \mathrm{PAr}-\mathrm{C}_{\mathrm{ipso}}\right)$, $134.96\left(\mathrm{~d}, 4 \mathrm{~J}(\mathrm{P}-\mathrm{C})=10.4 \mathrm{~Hz}, \mathrm{PAr}-\mathrm{C}_{\text {ortho }}\right), 134.62\left({ }^{2} \mathrm{~J}(\mathrm{P}-\mathrm{C})=\right.$ $2.0 \mathrm{~Hz}, \mathrm{Ar}-\mathrm{C}), 132.37,131.54,130.36$ (PAr-C para), 127.75 (d, 3) $\left.(\mathrm{P}-\mathrm{C})=11.2 \mathrm{~Hz}, \mathrm{PAr}-\mathrm{C}_{\text {meta }}\right), 123.34,121.64,80.4(\mathrm{OCH})$, $74.3\left(\mathrm{CH}_{2} \mathrm{~N}\right), 71.5\left(\mathrm{C}_{60}-\mathrm{Sp}^{3}\right), 63.1$ (OEt), 54.3, $51.9\left(\mathrm{NCH}_{3}\right), 14.1$
(OEt). ${ }^{31} \mathrm{P}\left\{{ }^{1} \mathrm{H}\right\} \mathrm{NMR}\left(\mathrm{CDCl}_{3}\right): \delta 17.5\left({ }^{1} \mathrm{~J}(\mathrm{Pt}-\mathrm{P})=4174 \mathrm{~Hz}\right)$. MALDI-TOF (9-NA): m/z $2170.1\left([\mathrm{M}-\mathrm{I}]^{+}\right)$. Anal. Calcd for $\mathrm{C}_{120} \mathrm{H}_{58} \mathrm{I}_{2} \mathrm{~N}_{2} \mathrm{O}_{4} \mathrm{P}_{2} \mathrm{Pt}_{2}$ : C 62.73, H 2.54, N 1.22. Found: C 62.59, $\mathrm{H}$ 2.46, N 1.18.

Crystal Structure Determinations. X-ray intensities were measured on a Nonius KappaCCD diffractometer with rotating anode ( $\mathrm{MoK} \alpha, \lambda=0.71073 \AA$ ) at a temperature of $150 \mathrm{~K}$ up to a maximal resolution of $\sin \vartheta / \lambda_{\max }=0.65 \AA^{-1}$. The structures were solved with automated Patterson methods with the program DIRDIF $97^{17}$ and refined with the program SHELXL $97^{18}$ against $F^{2}$ of all reflections. N on-hydrogen atoms were refined freely with ani sotropic displacement parameters; hydrogen atoms were refined as rigid groups. The drawings, structure calculations, and checking for higher symmetry were performed with the program PLATON. ${ }^{19}$

Structure 6: crystallized by diffusion of hexanes into a saturated solution of 6 in $\mathrm{CH}_{2} \mathrm{Cl}_{2}$. $\mathrm{C}_{55} \mathrm{H}_{54} \mathrm{Cl}_{2} \mathrm{~N}_{2} \mathrm{OP}_{2} \mathrm{Pt}_{2} \cdot \mathrm{CH}_{2} \mathrm{Cl}_{2}$, $\mathrm{fw}=1366.95$, colorless needle, $0.24 \times 0.18 \times 0.03 \mathrm{~mm}^{3}$, monoclinic, P2 $1 / c$ (No. 14), $a=12.9136(2) \AA, b=29.0033(4)$ $\AA, c=18.1500(2) \AA, \beta=129.378(1)^{\circ}, V=5254.58(12) \AA^{3}, Z=$ $4, \rho=1.728 \mathrm{~g} \mathrm{~cm}^{-3}, 39643$ measured reflections, 11898 unique reflections $\left(R_{\text {int }}=0.073\right.$ ). Absorption correction based on multiple measured reflections $\left(\mu=5.624 \mathrm{~mm}^{-1}, 0.59-0.99\right.$ transmission); 618 refined parameters, 0 restraints. The hydroxy and hydrogen substituents at C10 were refined with a disorder model (pseudo-2-fold rotation). $\mathrm{R}(\mathrm{I}>2 \sigma(\mathrm{I}))$ : $\mathrm{RI}=$ 0.0366 , wR2 $=0.0870$. $\mathrm{R}$ (all data): $\mathrm{R} 1=0.0476$, wR2 $=$ 0.0923. $\mathrm{S}=1.014$. Minimal and maximal residual electron density between -2.14 and $1.75 \mathrm{e} / \AA^{3}$.

Structure 10: crystallized by diffusion of $\mathrm{Et}_{2} \mathrm{O}$ into a saturated solution of $\mathbf{1 0}$ in $\mathrm{CH}_{2} \mathrm{Cl}_{2} . \mathrm{C}_{33} \mathrm{H}_{35} \mathrm{CINO}_{4} \mathrm{PPt}$, fw = 771.13, col orless needle, $0.54 \times 0.15 \times 0.09 \mathrm{~mm}^{3}$, orthorhombic, Pbcn (No. 60), $a=32.2580$ (2) $\AA, b=9.2442(1) \AA, c=20.8170-$ (1) $\AA, V=6207.62(8) \AA^{3}, Z=8, \rho=1.650 \mathrm{~g} \mathrm{~cm}^{-3}, 93074$ measured reflections, 7101 unique reflections $\left(R_{\text {int }}=0.068\right)$. Analytical absorption correction $\left(\mu=4.696 \mathrm{~mm}^{-1}, 0.13-0.70\right.$ transmission); 373 refined parameters, 0 restraints. $\mathrm{R}(\mathrm{I}>2 \sigma$ $(\mathrm{I})$ ): $\mathrm{R} 1=0.0222, \mathrm{wR} 2=0.0533$. $\mathrm{R}$ (all data): $\mathrm{R} 1=0.0271$, $w R 2=0.0553 . S=1.046$. Minimal and maximal residual electron density between -0.89 and $1.11 \mathrm{e} / \AA^{3}$.

Structure 13: crystallized by diffusion of $\mathrm{Et}_{2} \mathrm{O}$ into a saturated solution of 13 in $\mathrm{CH}_{2} \mathrm{Cl}_{2} . \mathrm{C}_{60} \mathrm{H}_{60}{ }_{2} \mathrm{~N}_{2} \mathrm{O}_{4} \mathrm{P}_{2} \mathrm{Pt}_{2}$, fw = 1579.02 , yellow needle, $0.18 \times 0.06 \times 0.03 \mathrm{~mm}^{3}$, monoclinic, $C 2 / c$ (No. 15), $a=30.3152(5) \AA, b=11.1579(2) \AA, c=18.7133-$ (3) $\AA, \beta=116.5565(7)^{\circ}, V=5662.01(17) \AA^{3}, Z=4, \rho=1.852$ $\mathrm{g} \mathrm{cm}^{-3}, 42384$ measured reflections, 6461 unique reflections $\left(R_{\text {int }}=0.070\right)$. Analytical absorption correction $(\mu=6.130$ $\mathrm{mm}^{-1}, 0.59-0.85$ transmission); 359 refined parameters, 3 restraints. The molecule has an exact, crystallographic $\mathrm{C}_{2}$ symmetry, leading to rotational disorder at carbon C10. R (I $>2 \sigma(\mathrm{I})): \mathrm{R} 1=0.0269, \mathrm{wR} 2=0.0524$. $\mathrm{R}$ (all data): $\mathrm{R} 1=$ $0.0374, w R 2=0.0554 . S=1.042$. Minimal and maximal residual electron density between -0.83 and $1.47 \mathrm{e} / \AA^{3}$.

Acknowledgment. This work was supported by the Council for Chemical Sciences of The Netherlands Organization for Scientific Research (CW-NWO).

Supporting Information Available: Tables of X-ray data for complexes $\mathbf{6 , 1 0}$, and $\mathbf{1 3}$. This material is available free of charge via the Internet at http://pubs.acs.org.

OM010500G

(17) Beurskens, P. T.; Admiraal, G.; Beurskens, G.; Bosman, W. P. Garcia-Granda, S.; Gould, R. O.; Smits, J. M. M.; Smykalla, C. The DIRDIF 97 program system, Technical Report of the Crystallography Laboratory; University of Nijmegen: The Netherlands, 1997.

(18) Sheldrick, G. M. SHELXL-97, Program for crystal structure refinement; University of Göttingen, Germany, 1997.

(19) Spek, A. L. PLATON. A multipurpose crystallographic tool Utrecht University: The Netherlands, 2000. 